\title{
Studies of dimension-six EFT effects in vector boson scattering
}

\author{
Raquel Gomez-Ambrosio ${ }^{\mathrm{a}}$ (D) \\ Institute for Particle Physics Phenomenology, Durham University, Durham DH1 3LE, UK
}

Received: 4 October 2018 / Accepted: 18 April 2019 / Published online: 6 May 2019

(C) The Author(s) 2019

\begin{abstract}
We discuss the implications of dimension-six operators of the effective field theory framework in the study of vector boson scattering in the $p p \rightarrow Z Z j j$ channel. We show that operators of dimension six should not be neglected in favour of those of dimension eight. We observe that this process is very sensitive to some of the operators commonly fit using LEP and Higgs data, and that it can be used to improve the bounds on the former. Further we show that other operators than the ones generating anomalous triple and quartic gauge couplings (aTGCs/aQCGs) can have a nonnegligible impact on the total and differential rates and their shapes. For this reason, a correct interpretation of the experimental results can only be achieved by including all the relevant bosonic and fermionic operators; we finally discuss how such an interpretation of experimental measurements can be developed.
\end{abstract}

\section{Contents}

1 Introduction . . . . . . . . . . . . . 1

2 SMEFT: notations and conventions . . . . . . 2

2.1 SMEFT amplitudes and cross sections . . . . . 3

2.2 Off-shell effects . . . . . . . . . . . . . . 4

2.3 VBS: Definition of the process . . . . . . . . 4

3 Weak-boson triple and quartic couplings ... . . 5

3.1 Anomalous couplings . . . . . . . . . . 5 5

3.2 SMEFT for triple and quartic gauge couplings . 5

3.3 Subsets of operators and gauge invariance . . . 6

4 EFT for the gauge couplings . . . . . . . . . . 6

4.1 Comparison with LEP and Higgs bounds . . . . 6

4.2 Linear and quadratic contributions . . . . . . . 7

5 The Warsaw basis in VBS . . . . . . . . . . . . 8

5.1 EFT for the full process . . . . . . . . . . 8

5.2 Four-fermion operators . . . . . . . . . 10

6 Di-jet observables . . . . . . . . . . . 11

a e-mail: raquel.gomez-ambrosio@durham.ac.uk
7 EFT in the background . . . . . . . . . . 11

7.1 Characterisation of the background . . . . . 11

8 General strategy for EFT studies at LHC . . . . . 13

9 Conclusions ................ . . 15

References . . . . . . . . . . . . . . . 15

\section{Introduction}

Effective field theories (EFTs) have become extremely popular in the last few years [1-36], proving to be a robust tool for New Physics (NP) searches and BSM physics studies. In general, one can use EFT to find the low-energy behaviour of a given UV theory (see for example Refs. [37-39]). Alternatively, one can use EFT in an almost model-independent way: as a generalised SM extension that can be used to parametrise small deviations observed on experimental measurements of SM observables. The latter is known as the "bottomup" approach and will be the one used in this work. The main underlying idea of the bottom-up approach is to add higher-dimensional operators to the (dimension-four) Standard Model Lagrangian, in a way that is consistent with the known symmetries: $S U(2) \times S U(3) \times U(1)$. A variety of relevant early work in this direction can be found in Refs. [40-45] and some interesting reviews on the topic are Refs. [46-48]. By adding new higher-dimensional terms to the SM Lagrangian, it is possible to parametrise small deviations from the original SM prediction. If such small deviations are found in the experimental data, it is possible to start mapping the space of "New Physics directions", ruling out some and focussing on others. While few questions remain unanswered in the current picture of fundamental interactions, some seem to be quite far from an answer: How can gravity be put on the same footing as the other fundamental interactions? What are dark matter and dark energy? Other important questions, however, can be tackled at LHC through precision tests of the SM. The most important ones concern the origin of electroweak symmetry breaking (EWSB). The 
Higgs mechanism [49-53] has been shown to give a very good description of EWSB, but some details of the latter are still unknown; for example, the fact that the spontaneous symmetry breaking can be realised in linear or non-linear representations. The answer to this enigma may lie in the gauge couplings, which have only been partially studied at LEP: triple gauge couplings have only been observed in a very concrete energy regime and under a set of assumptions regarding the final-state radiation whereas the interactions between four gauge bosons will only be observed at the LHC.

To address the last question, the detailed study of the Vector Boson Scattering (VBS) process is essential. This process is characterised at tree level by the exchange of weak gauge bosons between two quarks or a quark and an antiquark, which gives us direct access to both triple and quartic gauge couplings. Since this is a purely electroweak process, it has a relatively small cross-section at LHC where the large QCD backgrounds dominate everywhere. However, the family of VBS processes has very particular experimental signatures: two very energetic forward jets with a big rapidity gap between them. In this work we combine these two interesting fields, by applying EFT techniques to the study of VBS at LHC. In particular, we study the effects of different dimension-six ( $\operatorname{dim}=6$ ) operators in the total cross section and differential distributions of the purely electroweak contribution to the process $p p \rightarrow Z Z j j$, more commonly known as $\operatorname{VBS}(Z Z)$. We perform a numerical study of the aforementioned quantities, leaving a complete analytical description for future study.

The outline of the paper is as follows: in Sect. 2 we introduce our notation and conventions as well and define of the family of VBS processes. In Sect. 3 we summarise the state of the art, both on theoretical and experimental aspects, and we discuss the issue of anomalous couplings. In Sect. 4.1 we compare some of the published results for dimension-six operator fits with our predictions for the cross section of this process. In Sects. 4 and 5 we study the impact of different operators of the Warsaw basis on the differential distributions. At first, we focus only on TGC/QGC operators. The full analysis is shown in Sect. 5.1, where we take into account all the Warsaw-basis operators, bosonic and fermionic. In Sect. 6 we focus on the main signatures for VBS: di-jet observables. Showing that the effects of certain operators (in particular the four-quark ones) can be enhanced on such observables. As anticipated, the family of VBS processes have a relatively small cross section at LHC. The situation for the $Z Z$ final state is particularly dramatic, where the QCD background is very large, with a signal to background ratio of up to $1 / 20$ in some phase space regions. For this reason, a rigorous treatment of the process demands also the study of the EFT effects on the corresponding background, which we perform in Sect. 7. Finally, in Sect. 8, we discuss a possible strategy for a global analysis including all the dimension-six operators relevant to this process.

\section{SMEFT: notations and conventions}

In this work, the bottom-up approach to EFT is used. The SM Lagrangian is extended with higher-dimensional operators consistent with the known SM symmetries. Furthermore, we assume a linear representation for the physical Higgs field, in the form an SU(2) doublet. Such a theory is commonly known as SMEFT:

$$
\begin{aligned}
\mathcal{L}_{\mathrm{SMEFT}}= & \mathcal{L}_{\mathrm{SM}}+\frac{c^{(5)}}{\Lambda} \mathcal{O}^{(5)}+\frac{1}{\Lambda^{2}} \sum_{i} c_{i}^{(6)} \mathcal{O}_{i}^{(6)} \\
& +\sum_{j} \sum_{k} \frac{1}{\Lambda^{2+k}} c_{j}^{(6+k)} \mathcal{O}_{j}^{(6+k)} .
\end{aligned}
$$

At dimension five, $\operatorname{dim}=5$, there is only one possible operator, from Ref. [54], which does not enter the process studied in this work. At $\operatorname{dim}=6$, the complete basis has 59 operators in the flavour universal case and 2499 in the most general case. In this work we will use a parametrisation of the former, commonly known as the Warsaw basis [55].

A general method to construct higher-dimensional bases using Hilbert series was proposed in Ref. [56]. In the context of VBS, some subsets of $\operatorname{dim}=8$ operators affecting quartic gauge couplings have been proposed in Refs. [57,58].

Other EFT bases There are additional dimension-six bases, other than the Warsaw basis. It is quite common to use the SILH basis, from Ref. [1] in Higgs phenomenology; however, it is not optimised for multiboson processes. Instead, there is a VBS-dedicated basis, typically known as the HISZ basis, from Ref. [59].

Parameter shifts Adding higher-dimensional terms to the SM Lagrangian has three consequences: firstly, new vertices appear such as those with four fermions. Secondly, the SM vertices get modified with an additional EFT contribution of the form $V_{\mathrm{SM}}=a \cdot g+b \cdot g \cdot c_{i} / \Lambda^{2}$, where $g$ is the SM coupling and $c_{i}$ is the Wilson coefficient associated with the $i$ th $\operatorname{dim}=$ 6 operator. Thirdly, there are shifts on the other SM parameters: the masses, vev, the Weinberg mixing angle and gaugefixing parameters. For a detailed discussion of the parameter shifts and gauge fixing in SMEFT see Refs. [7,60-62].

The easiest example to understand parameter shifts is that of the Higgs field: if we add the Warsaw-basis operators to the SM Lagrangian, the Higgs part of the Lagrangian becomes

$$
\begin{aligned}
\mathcal{L}_{\text {Higgs,EFT }}= & \partial_{\mu} \Phi^{\dagger} \partial^{\mu} \Phi-\lambda\left(\Phi^{\dagger} \Phi-\frac{v^{2}}{2}\right) \\
& +\frac{c_{\mathrm{H}}}{\Lambda^{2}} \mathcal{O}_{\mathrm{H}}+\frac{c_{H} \square}{\Lambda^{2}} \mathcal{O}_{\mathrm{H} \square}+\frac{c_{H D}}{\Lambda^{2}} \mathcal{O}_{\mathrm{HD}},
\end{aligned}
$$


where:

- $\mathcal{O}_{\mathrm{H}}=\left(\Phi^{\dagger} \Phi\right)^{3}$,

- $\mathcal{O}_{\mathrm{HD}}=\left(\Phi^{\dagger} D_{\mu} \Phi\right)^{*}\left(\Phi^{\dagger} D^{\mu} \Phi\right)$,

- $\mathcal{O}_{H \square}=\left(\Phi^{\dagger} \Phi\right) \square\left(\Phi^{\dagger} \Phi\right)$,

and $\Phi$ is the Higgs doublet,

$\Phi=\frac{1}{\sqrt{2}}\left(\begin{array}{c}h+v+i \phi^{0} \\ \sqrt{2} i \phi^{-}\end{array}\right) \Rightarrow \Phi=\frac{1}{\sqrt{2}}\left(\begin{array}{c}h+v \\ 0\end{array}\right)$,

in the Feynman and unitary gauges, respectively. By expanding Eq. (2.2) we see that the kinetic term is modified as well as the potential (i.e. its minimum gets shifted). To restore the correct vacuum expectation value and canonical normalisation of the kinetic term, the corresponding SM parameter and field have to be redefined as

$$
\begin{aligned}
v & \rightarrow v\left(1+\Delta_{6}(v)\right), \quad h \rightarrow h\left(1+\Delta_{6}(h)\right), \\
\phi^{0} & \rightarrow \phi^{0}\left(1+\Delta_{6}\left(\phi^{0}\right)\right) .
\end{aligned}
$$

After some simple algebra one finds

$$
\begin{aligned}
v & \rightarrow v\left(1+\frac{3 v^{2}}{8 \lambda} \frac{c_{\mathrm{H}}}{\Lambda^{2}}\right), \\
h & \rightarrow h\left(1+\frac{v^{2}}{\Lambda^{2}} c_{\mathrm{H} \square}-\frac{v^{2}}{\Lambda^{2}} \frac{c_{\mathrm{HD}}}{4}\right), \\
\phi^{0} & \rightarrow \phi^{0}\left(1-\frac{v^{2}}{\Lambda^{2}} \frac{c_{\mathrm{HD}}}{4}\right) .
\end{aligned}
$$

As is customary in the literature, we redefine the Wilson coefficients as $\bar{c}_{i}=\frac{v^{2}}{\Lambda^{2}} c_{i}$.

The consequence of the field and parameter redefinitions in Eq. (2.5) is that each and every vertex containing the Higgs field will be dependent on the Wilson coefficients $\left\{c_{\mathrm{H}}, c_{\mathrm{HD}}\right\}$. This effect is nothing else than a wave-function renormalisation in the more classical sense.

The same phenomenon occurs for all the other fields and parameters. The case of the EW sector has to be handled with great care, especially when working on the Feynman gauge since new linear transitions between gauge and Goldstone bosons appear. The gauge sector of the Lagrangian becomes

$$
\begin{aligned}
\mathcal{L}_{\text {Gauge }, \text { SMEFT }}= & \mathcal{L}_{\text {Gauge }, \text { SM }}+\frac{c_{\mathrm{HB}}}{\Lambda^{2}} \mathcal{O}_{\mathrm{HB}}+\frac{c_{\mathrm{HW}}}{\Lambda^{2}} \mathcal{O}_{\mathrm{HW}} \\
& +\frac{c_{\mathrm{HWB}}}{\Lambda^{2}} \mathcal{O}_{\mathrm{HWB}}+\frac{c_{\mathrm{HG}}}{\Lambda^{2}} \mathcal{O}_{\mathrm{HG}} .
\end{aligned}
$$

As a consequence, the gauge fields get shifted in the same fashion as shown for the Higgs field. The neutral gauge boson for example ${ }^{1}$ :

\footnotetext{
1 There are many different ways to redefine the fields, depending on the gauge-fixing procedure chosen [60-62], and the treatment of the $Z-A$ transition. Equation (2.7) represents only one option.
}

$$
\begin{aligned}
Z_{\mu} \rightarrow Z_{\mu} & \left(1+\frac{v^{2}}{\Lambda^{2}}\left\{\sin \left(\theta_{w}\right)^{2} C_{\mathrm{HB}}+\cos \left(\theta_{w}\right)^{2}\right.\right. \\
& \left.\left.+C_{\mathrm{HWB}} \sin \left(\theta_{w}\right) \cdot \cos \left(\theta_{w}\right)\right\}\right),
\end{aligned}
$$

where $\theta_{w}$ is the Weinberg mixing angle.

For this reason, when one studies a concrete process, it is also important to take into account these shifts and not only the EFT effects on single vertices. For example, the operator $\mathcal{O}_{\mathrm{HB}}$ does not directly modify triple or quartic gauge vertices, but it enters the $Z$ field normalisation and hence any vertex containing the former.

In addition to the fields, the parameters $\left\{M_{\mathrm{W}}, M_{\mathrm{z}}, \theta_{w}\right.$, $\left.g, G_{\mathrm{F}}\right\}$ are also shifted. It is well known that these parameters are not all independent. The relation $M_{\mathrm{Z}}=\frac{M_{\mathrm{W}}}{\cos \left(\theta_{w}\right)}$, is valid at leading order in the SM, but has to be corrected at NLO. The same holds for the SMEFT, where this and similar relations need to be re-examined (e.g $\left.\theta_{w}\left(g, g^{\prime}\right), M_{\mathrm{H}}\left(\mathrm{G}_{\mathrm{F}}, v\right)\right)$. For this reason, the input parameter set chosen for a calculation will involve different EFT parameters depending on the choice.

Importance of the input parameter set (IPS) It is important to recall that different IPSs lead to different predictions, already at tree level. While the " $\alpha$-scheme": $\left\{M_{\mathrm{Z}}, \alpha, \mathrm{G}_{\mathrm{F}}\right\}$ might be convenient at lower energies, or when using experimental measurements of EWPD, for the energy scale of our process $\left(E \approx 2 M_{\mathrm{Z}}\right)$ the " $M_{\mathrm{W}}$-scheme", $\left\{M_{\mathrm{z}}, M_{\mathrm{W}}, \mathrm{G}_{\mathrm{F}}\right\}$, is a better choice. The modifications to the vev are shown in Eq. (2.5), and analogously, $\mathrm{G}_{\mathrm{F}}$ gets modified by the $\left\{c_{\ell \ell}, c_{H l}^{(3)}\right\}$ operators. The one-loop renormalisation of $\mathrm{G}_{\mathrm{F}}$ in SMEFT has very recently been calculated in Ref. [63].

The Wilson coefficients of the effective theory are not observable quantities, and the $\overline{\mathrm{MS}}$ renormalisation scheme is adopted. The SM masses and the electroweak coupling, on the other hand, can be related to experimental quantities and the on-shell renormalisation scheme can be adopted.

\subsection{SMEFT amplitudes and cross sections}

In order to derive physical quantities, it is convenient to look further than the EFT Lagrangian, to $S$-matrix elements, since those are the gauge invariant objects that can be projected into experimental quantities. The most general EFT amplitude can be expressed as

$$
\begin{aligned}
\mathcal{A}_{\mathrm{EFT}}= & \mathcal{A}_{\mathrm{SM}}+\bar{g} \mathcal{A}_{6}^{(1,1)}+\bar{g}^{2} \mathcal{A}_{6}^{(1,2)}+\frac{\bar{g}}{(4 \pi)^{2}} \mathcal{A}_{6}^{(2,1)} \\
& +\frac{\bar{g}^{2}}{(4 \pi)^{2}} \mathcal{A}_{6}^{(2,2)}+\frac{\bar{g}}{\Lambda^{2}} \mathcal{A}_{8}^{(1,1)}+\cdots
\end{aligned}
$$

where $\bar{g}=g / \Lambda^{2}$. The first EFT term corresponds to one $\operatorname{dim}=6$ insertion in the original tree-level diagram, the second term represents two dim $=6$ insertions, then one insertion and one loop, two insertions and one loop, then one 

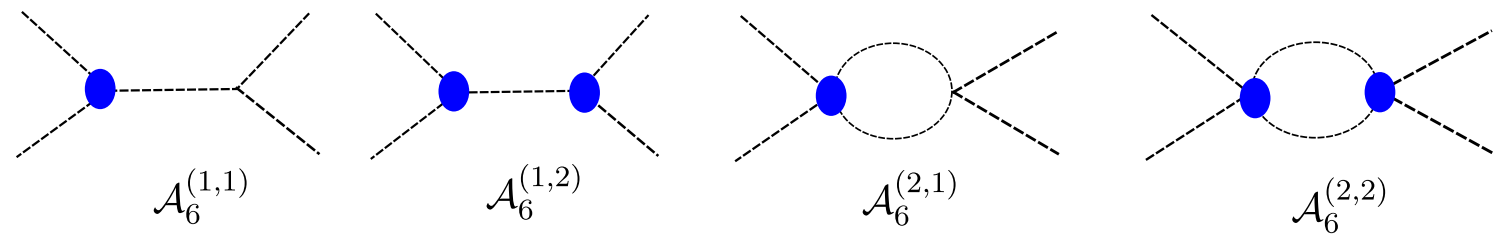

Fig. 1 Perturbative expansion of the EFT dim $=6$ amplitudes, illustrating Eq. (2.8)

$\operatorname{dim}=8$ insertion at tree level and so on, as illustrated in Fig. 1.

The leading-order EFT corresponds to the first term, $\mathcal{A}_{6}^{(1,1)}$. The next term, $\mathcal{A}_{6}^{(1,2)}$, with two insertions at tree level, is tricky, since it is of order $\left(1 / \Lambda^{2}\right)^{2}$ and the EFT expansion is only order by order renormalisable in the expansion parameter $1 / \Lambda$. For this reason, it is normally not included in the EFT nor in the NLO-EFT calculations, unless the corresponding counterterms are available. The term $\mathcal{A}_{6}^{(2,1)}$ accounts for the NLO-EFT amplitude, with one-loop-one-insertion diagrams.

The perturbative EFT expansion, then, grows in different directions: higher-dimensional operators or higher loops. Whether the tree-level dim $=8$ correction is larger than the NLO-dim $=6$ term will depend on the energy of the next new physics scale $\Lambda$ which, up to now, is unknown. In this work, the leading order for an EFT amplitude is defined as $\mathcal{A}=\mathcal{A}_{\mathrm{SM}}+g_{6} \mathcal{A}_{6}^{(1,1)}$. Still, some ambiguities appear when squaring the amplitude in (2.8),

$$
\begin{aligned}
\left|\mathcal{A}_{\mathrm{EFT}}\right|^{2}= & \left|\mathcal{A}_{\mathrm{SM}}\right|^{2}+\bar{g}\left|\mathcal{A}_{\mathrm{SM}} \cdot \mathcal{A}_{6}^{(1,1)}\right|+\bar{g}^{2}\left|\mathcal{A}_{6}^{(1,1)}\right|^{2} \\
& +\bar{g}^{2}\left|\mathcal{A}_{\mathrm{SM}} \cdot \mathcal{A}_{6}^{(1,2)}\right|+\frac{\bar{g}}{\Lambda^{2}}\left|\mathcal{A}_{\mathrm{SM}} \cdot \mathcal{A}_{8}^{(1,1)}\right|+\cdots
\end{aligned}
$$

The term $\sim \bar{g}^{2}\left|\mathcal{A}_{6}^{(1,1)}\right|^{2}$, is commonly called "quadratic EFT" in the literature. It can be used as an estimate for the theoretical uncertainty; it is interesting to study it in depth too, since it is positive definite and hence it can have relevant effects on the differential distributions and on the unitarity bounds of the EFT expansion. This term will be studied in the context of VBS in Sect. 4.2.

\subsection{Off-shell effects}

Higher-dimensional operators are always suppressed by a power of the NP cut-off, $\Lambda$. This means that the expansion parameter in the perturbative EFT expansion is $E / \Lambda$, where $E$ is the energy scale of the process under study ${ }^{2}$, i.e. near the $Z$-pole we can think of the EFT expansion in terms of $M_{\mathrm{z}}^{2} / \Lambda^{2}$ whereas, away from the peak, the EFT effects are

\footnotetext{
2 This interpretation is consistent with the fact that the EFT coefficients $c_{i}$ run with the energy scale, just as every other Lagrangian parameter.
}

more accurately parametrised by $p_{\mathrm{T}}^{2} / \Lambda^{2}$. For this reason, the high-energy regions (tails of the $p_{\mathrm{T}}$ distributions) are the ones where the EFT effects are expected to be largest (i.e. $E_{1} / \Lambda \gg E_{2} / \Lambda$, for $E_{2}$ on the pole and $E_{1}$ on the tail).

The VBS process is defined by two very energetic jets. This means that the $p_{\mathrm{T}}(j)$ and $m_{j j}$ distributions are privileged kinematic variables as regards where to expect EFT effects.

\subsection{VBS: Definition of the process}

The family of vector boson scattering processes is very interesting, since it lies at the heart of electroweak symmetry breaking. Some work describing the details of these processes is in Refs. [64-68]. Unitarity and gauge invariance are conserved in this process thanks to a series of cancellations between Feynman diagrams, and fundamentally thanks to the introduction of the Higgs boson, see Ref. [69,70]. For these reasons, VBS represents a set of privileged channels for NP studies. For some applications of NP searches to VBS see for example Refs. [71-79].

There are many possible definitions of the VBS process. Typically, there are substantial differences between the theoretical definitions, in terms of initial and final states, and the experimental definitions, which constrain the phase space of the final states as well. In particular, it is common that the experimental analyses impose certain cuts to try and decouple the vector boson fusion (VBF) process, where a Higgs boson in the s-channel is produced from the exchange of weak bosons between a quark and an antiquark. This way, the VBF channel is studied in dedicated Higgs analyses, whereas the VBS channels belong to the multiboson analyses.

Regarding such cuts, there are different VBS regions that are widely accepted, but they obviously lead to different results. In the most recent LHC results, in Ref. [80], the $\operatorname{VBS}(\mathrm{ZZ})$ is defined as the purely electroweak component of $p p \rightarrow Z Z j j \rightarrow \ell \bar{\ell} \ell^{\prime} \bar{\ell}^{\prime} j j$, measured in the region defined by the following cuts:

$$
\begin{aligned}
& \text { - } p_{\mathrm{T}}(j)>30 \mathrm{GeV} \text {, } \\
& \text { - } m_{j j}>100 \mathrm{GeV} .
\end{aligned}
$$

One can define a VBS-enriched region, with the additional cuts: 
- $\Delta \eta\left(j_{1} j_{2}\right)>2.4$

- $m_{j j}>400 \mathrm{GeV}$,

which was also used in some parts of that analysis. In this work we chose a compromise between both regions, and apply the cuts:

- $p_{\mathrm{T}}(j)>30 \mathrm{GeV}$,

- $\Delta \eta\left(j_{1} j_{2}\right)>2.4$,

- $m_{j j}>100 \mathrm{GeV}$.

As anticipated, further cuts impose the requirement that two $Z$ bosons be on-shell, to remove the VBF contamination. Nevertheless it is important to keep in mind that the experimental cut defining "on-shell" $Z$ 's $\left(M_{\ell \bar{\ell}} \in[60,120] \mathrm{GeV}\right)$ is not strictly the same thing as the theory definition for the $Z$ pole.

As a matter of clarity, in this work we focus only on the process $p p \rightarrow Z Z j j$ before the on-shell decay. The difference between the process $p p \rightarrow Z Z j j$ followed by the on-shell decay $Z \rightarrow \ell \bar{\ell}$ and the process $p p \rightarrow \ell \bar{\ell} \ell^{\prime} \bar{\ell}^{\prime} j j$ in the aforementioned fiducial region is negligible. In general, when applying multivariate analysis techniques the first definition is preferred, since it populates the phase space in a more effective way.

For a rigorous EFT treatment the same study should be performed including the decays in order to make sure that the difference between the two options is also negligible in SMEFT. It is clear that new operators will come into play, mainly the ones connecting quarks and leptons in the final state; however, intuition and experience tell us that the VBS cuts will most likely remove the bulk of that contribution.

\section{Weak-boson triple and quartic couplings}

\subsection{Anomalous couplings}

Anomalous gauge couplings were introduced in Ref. [81], at a time when the EWSB mechanism had not been thoroughly tested, and before the Higgs boson was discovered. Such couplings, defined in terms of ad hoc variations on the Lagrangian parameters, might be good in a first approximation, but they present serious theoretical inconsistencies. The main problem is that they violate gauge invariance and unitarity beyond the leading order.

The EFT approach aims to parametrise small deviations from the SM predictions, which are currently being tested with unprecedented precision at LHC. In that regard, a more consistent approach to anomalous couplings is needed. In particular, one that is consistent at next-to-leading order. The SMEFT approach considered in this work, in terms of dimension-six operators, represents an optimal solution to the anomalous coupling problem: it can be understood as a SM Lagrangian where all the parameters are anomalous, but in a way consistent with the QFT rules.

Numerous works regarding anomalous gauge couplings in SMEFT can be found in the literature. Some or the earliest studies are Refs. [59,82-85], and more current ones can be found for example in Refs. [86-91]. In the upcoming sections we will investigate and discuss the differences between allowing the EFT operators only on the weak-couplings (in the spirit of the anomalous coupling approach) or allowing them to occur anywhere.

\subsection{SMEFT for triple and quartic gauge couplings}

As part of the legacy of the LEP experiment, it is common in the experimental analysis to study triple gauge couplings (TGCs) in multiboson production channels. For some LEP/LEP2 results, see Refs. [92-96], and for some LHC analyses, see Refs. [97-99]. Quartic gauge couplings (QCGs), on the contrary, were more difficult to access at LEP/LEP2 (for example in $e^{+} e^{-} \rightarrow \gamma \gamma \nu \bar{\nu}$ and $e^{+} e^{-} \rightarrow$ $\gamma \gamma q \bar{q}$ were studied in Refs. $[100,101])$ and not all QGCs where accessible.

This fact makes the QGCs an interesting goal for the LHC experiments, where QGCs are studied in the VBS channels, for example in the analysis of Ref. [80]. Still, it is important to emphasise that this approach is extremely misleading, since it implies identifying a collection of thousands of Feynman diagrams with a single (off-shell!) vertex, and more importantly, it implies the assumption that triple and quartic gauge couplings do not originate simultaneously through the EWSB. For this reason, an analysis in terms of cross sections, differential distributions or (pseudo-)observables should always be preferred.

At the LEP experiment, the $s$-channel production of $Z / W$ bosons was very well under control, as well as their decays, since the electroweak radiation could be deconvoluted pretty accurately from the process. This made it possible to treat TGCs as pseudo-observables, which could be measured by the experiments. This is not the case any more at the LHC, and hence one should not aim at "measuring" triple or quartic electroweak couplings.

Pseudo-observables are well-defined theoretical quantities that can be measured in the experiment; a classic example is that of the set of EWPDs. The most promising alternative for LHC physics relies on the study of the residues of S-matrix poles, which are by default gauge invariant quantities. For some work in this direction, see Refs. [102-106], and the reviews $[47,107]$.

The impact of dim $=6$ operators on triple gauge couplings has been studied in Refs. [88,89]. The set of dim $=8$ operators affecting quartic gauge couplings has been studied in depth in Refs. $[57,58,108]$, and another dim $=8$ sub- 
set, relevant for diboson studies, has been addressed in Ref. [109]. Similar studies to the one presented here, tackling vector boson scattering in the $\operatorname{dim}=8$ basis have been presented in Refs. [110,111], as so has work on VBS in the context of the electroweak chiral Lagrangian in Refs. [112,113]. However, there is no study, to the best of our knowledge, addressing $\operatorname{dim}=6$ effects in the context of VBS.

\subsection{Subsets of operators and gauge invariance}

Each of the operators in the Warsaw basis is independently gauge invariant and in principle it is possible in a treelevel study to select a subset of operators without breaking this gauge invariance. However, this situation will not hold beyond tree level, where different operators enter through the $\operatorname{dim}=6$ counterterms, and the full basis is needed for UV-renormalisation. For a further discussion of the renormalisation of SMEFT see Refs. [7,8,10,60].

Gauge invariance is also broken if the effects of certain $\operatorname{dim}=6$ or $\operatorname{dim}=8$ operators are only included on a certain vertex and not in other vertices or wave-function normalisations. For example, it is gauge invariant to include only $\mathcal{O}_{W}$, $\mathcal{O}_{\text {HW }}$ and $\mathcal{O}_{\text {HWB }}$, neglecting $\mathcal{O}_{\text {HB }}$. But it is not completely rigorous: $\mathcal{O}_{\mathrm{HB}}$ enters every vertex containing a $Z$ field, as shown in Eq. (2.7), and every expression containing the Weinberg mixing angle, and it might enter in different ways depending on the IPS chosen. The same holds for other operators, mainly $\mathcal{O}_{\ell \ell}$ and $\mathcal{O}_{\mathrm{Hl}}^{(3)}$, that enter as corrections to $\mathrm{G}_{\mathrm{F}}$.

\section{EFT for the gauge couplings}

To allow a straightforward comparison to be made with the existing literature, in this section we study the impact of a handful of EFT operators. In particular, we study the operators that directly affect triple and quartic gauge vertices, and that are $\mathrm{CP}$-even. ${ }^{3}$ We have:

- $\mathcal{O}_{\mathrm{W}}=\epsilon^{i j k} W_{\mu}^{i v} W_{\nu}^{j \rho} W_{\rho}^{k \mu}$,

- $\mathcal{O}_{\mathrm{HW}}=H^{\dagger} H W_{\mu \nu}^{I} W^{\mu \nu I}$,

- $\mathcal{O}_{\mathrm{HWB}}=H^{\dagger} \tau^{I} H W_{\mu \nu}^{I} B^{\mu \nu}$.

For this preliminary study we generated $3 \times 10^{5}$ events for the process defined in Sect. 2.3 using a modified version of the SMEFTSim package [105], interfaced with Madgraph5_aMC@NLO [114] via FeynRules [115] and MadAnalysis5 [116]. We studied the impact of each of the three TGC/QGC operators separately, as well as the sum of them, following the definition of leading-order EFT given in Sect. 2.1.

\footnotetext{
3 The analysis can easily be extended to the CP-odd case, by adding $\mathcal{O}_{\widetilde{\mathrm{W}}}, \mathcal{O}_{\widetilde{\mathrm{HW}}}$ and $\mathcal{O}_{\widetilde{\mathrm{HWB}}}$.
}

In Fig. 2 we see the impact of each of the three operators individually and the sum of them, for four different observables: the invariant mass of the two final $Z$ and that of the two final jets, and the transverse momentum of the leading $Z$ and leading jet. For the numerical values of the coefficients, in this section, we choose the democratic values $\bar{c}_{W}=\bar{c}_{\mathrm{HW}}=\bar{c}_{\mathrm{HWB}}=0.06$, which correspond to $c_{W}=c_{\mathrm{HW}}=c_{\mathrm{HWB}}=1$ with $\Lambda=1 \mathrm{TeV}$. In Sect. 4.1 we will discuss the case of the available best-fit values.

We observe that the EFT effects on the invariant mass distributions are relatively homogeneous, in particular in the two-jet case. The VBS signature is characterised by two very energetic jets and the high-energy phase space is quite well populated. On the contrary, for the $Z Z$ invariant mass we find that at very high energies we reach the limit where the SM production tends to zero, and the EFT effects become sizeable. This effect is, of course, dominated by the Monte Carlo uncertainty, but it points us to a region that should be studied in detail. Such regions where the SM production becomes negligible are also those where the quadratic EFT of Eq. (2.9) will have a dominant role. This will be discussed in Sect. 4.2.

The cases of transverse momentum distributions are very interesting themselves. We find that the EFT effects get enhanced on the tails of such distributions, which is something that we would have expected a priori for all four observables, but is not so pronounced as expected for the invariant masses. For the two cases $p_{T}\left(Z_{1}\right)$ and $p_{T}\left(j_{1}\right)$ we also reach the regime where the SM production is negligible but the EFT effects remain.

\subsection{Comparison with LEP and Higgs bounds}

Several works have appeared in the last years, where SMEFT predictions are compared with LEP data (in Refs. [5,117, 118]) and LHC data (the SILH basis in Refs. [119,120] and more recently the Warsaw basis in Refs. [121-123]).

In order to be consistent, a global fit of the full Warsaw basis would be desirable. For this reason, it is necessary to include as many measurements as possible, including fiducial cross sections as well as differential distributions.

In this section we study how the published best-fit values enter the VBS(ZZ) fiducial cross section. In Fig. 3 we show the signal strength $\mu=\frac{\sigma_{\mathrm{EFT}}}{\sigma_{\mathrm{SM}}}$ for the central values given by the profile fit of the operators (black, points) and their $95 \%$ confidence level bounds (red, error bars). ${ }^{4}$

\footnotetext{
${ }^{4}$ The data are taken from the aforementioned papers. For the LEP fit we took the values corresponding to the $M_{\mathrm{W}}$ IPS and to a $1 \%$ theoretical uncertainty. For the Higgs fit we took the values in Table 4 of Ref. [122], but it was not possible to clarify such details (IPS and th. uncertainty) with the authors, these could be the origin of some of the large interference terms found.
} 

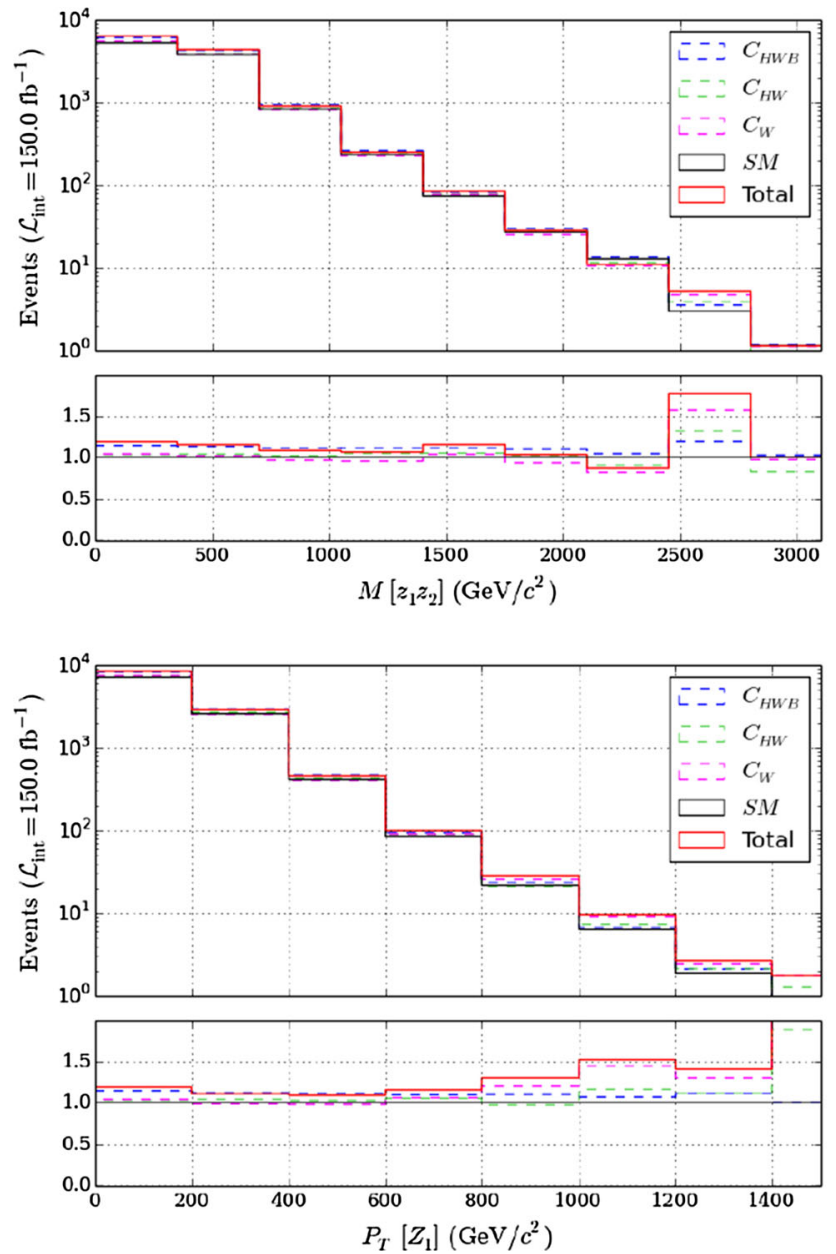

Fig. 2 Here we show the impact of the three Warsaw-basis operators that affect the triple and quartic gauge couplings. We set the values $\bar{c}=c \frac{v^{2}}{\Lambda^{2}}=0.06$, which correspond to $c=1$ for $\Lambda=1 \mathrm{TeV}$. However, it is important to recall that one of the main assumptions of the

For the LEP case, we find that the values for some operators are off by a large amount $(\geq 200 \%)$, which means that this channel could be an interesting one to constrain such a fit better. This is not surprising since, as precise as it was, LEP is still a "low-energy" experiment, ${ }^{5}$ compared with the energies considered here and throughout LHC Run-2. Moreover, the treatment of the radiation in LEP raises some doubts on the applicability of such measurements for EFT searches, as discussed in Sect. I.5 of Ref. [47].

In the LHC case, we find that the fit is more precise and its predictions are compatible with the ones here. The total contribution including its error bar, however, still departs from the SM expected value. Furthermore, it is important to recall

5 An important step of any EFT calculation requires the matching of the EFT calculation, at a low energy scale $E_{0}$, with the "new physics" scale $\Lambda$, to account for the running of the EFT coupling.
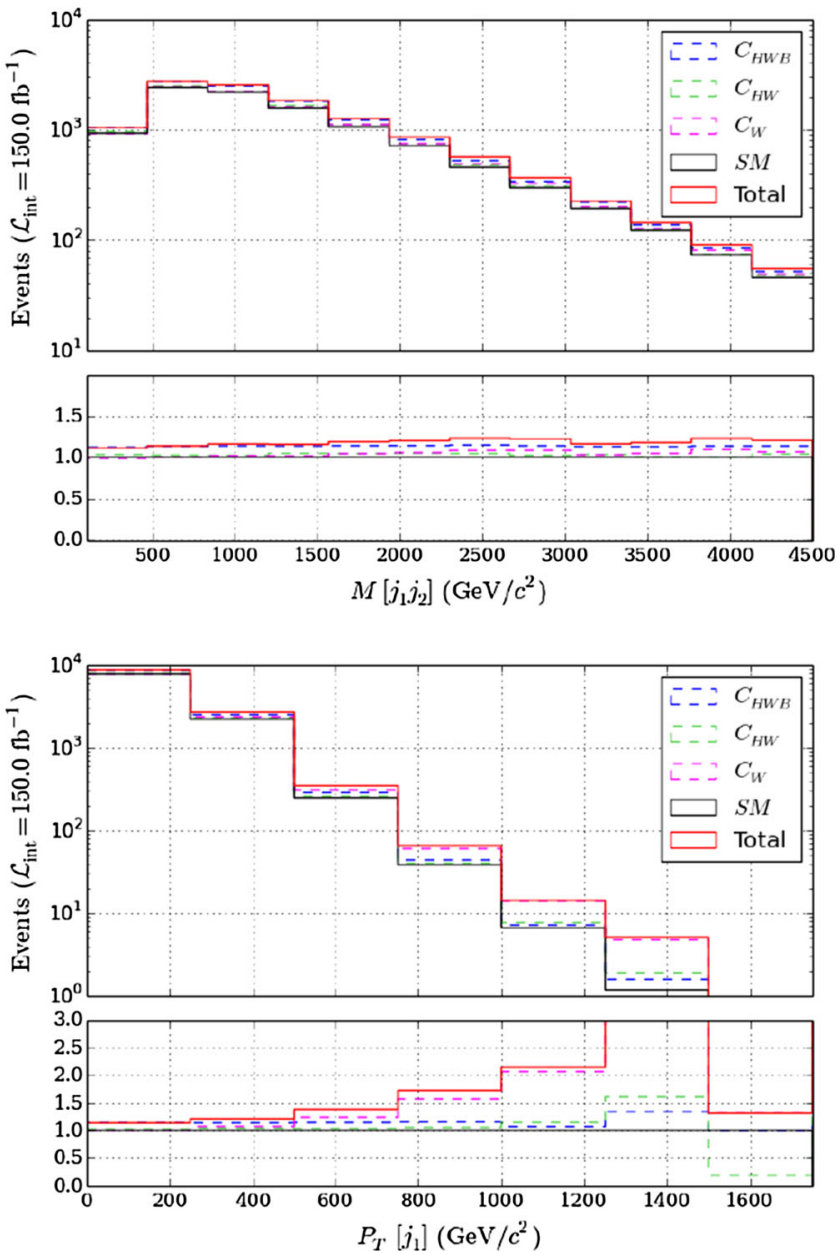

EFT is that there are no new light resonances, and hence in a histogram like this one we are implicitly assuming $\Lambda>3 \mathrm{TeV}$, while keeping $\bar{c}=c \frac{v^{2}}{\Lambda^{2}}=0.06$

that only a subset of EFT operators is included in the former fits, and hence some directions in the phase space have not been tested. The results shown on Fig. 3 further show that only one of the four-fermion operators $\left(\mathcal{O}_{\ell \ell}\right.$, the one entering $\mathrm{G}_{\mathrm{F}}$ ) is well constrained, whereas other 11 of these operators enter the VBS cross section. Contrary to the common belief that four-fermion operators are maximally constrained from LEP measurements, we see that, while this might be the case for most of the leptonic operators, it is not the case for fourquark ones.

\subsection{Linear and quadratic contributions}

Another issue that should be handled with care is the treatment of quadratic dim $=6$ terms. This effect was discussed in Sect. 2.1, and it was thoroughly studied in Sect. I.4.7 of Ref. [47] and in Refs. [124,125]. The quadratic contribution 


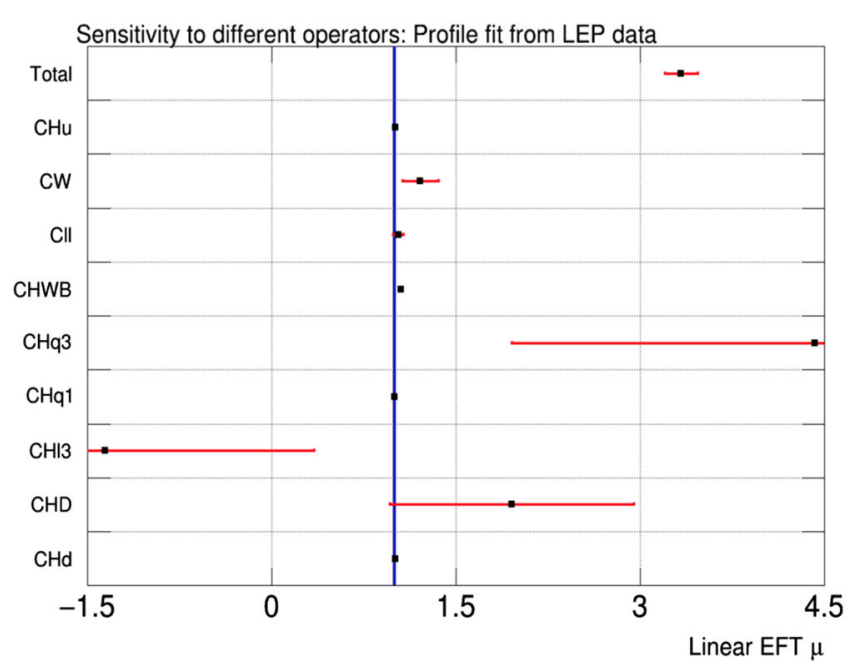

Fig. 3 Relative contribution given by each of the best-fit values. Operators that enter the VBS(ZZ) cross section but have not been fitted in the past have been set to zero in these figures. On the left we use the values from the LEP fit, Ref. [118] and on the right from the LHC fit Ref. [122]

to the cross section is not included in the SMEFT predictions, as a matter of consistency: its perturbative order is higher than the linear term, equivalent to the $\operatorname{dim}=8$ operators, which are not included either. However, this quadratic term is, by definition, always positive, and hence can have a large impact on the behaviour of the final distributions. There are some situations where the quadratic contribution should be carefully studied:

- In the regions when the SM prediction becomes very small. In that case the interference term $\mathrm{SM} \times \mathrm{EFT}_{6}$ is dominated by the EFT contribution, which is expected to be large. If this is the case, the quadratic term must also be quite large and should be calculated as part of the theoretical uncertainty.

- In the cases where the linear $\operatorname{dim}=8$ interference term is included $\left(\mathrm{SM} \times \mathrm{EFT}_{8}\right)$, since they are both of the same perturbative order, $\mathcal{O}\left(\Lambda^{-} 4\right)$.

The latter situation, where dim $=8$ operators where compared with $\operatorname{dim}=6$ quadratic terms, was recently studied in Ref. [126]. In Fig. 4 we show an example of the impact of including the quadratic contribution in some key distributions, for the canonical value $\Lambda=1 \mathrm{TeV}$. Some good news is that, for higher values of the New Physics scale, $\Lambda$, the ratio between quadratic and linear contributions is expected to be less pronounced. That is, $r=\frac{\left(1 / \Lambda^{2}\right)}{(1 / \Lambda)}=\frac{1}{\Lambda}$, hence $r_{2} \ll r_{1}$ for $\Lambda_{2} \gg \Lambda_{1}$

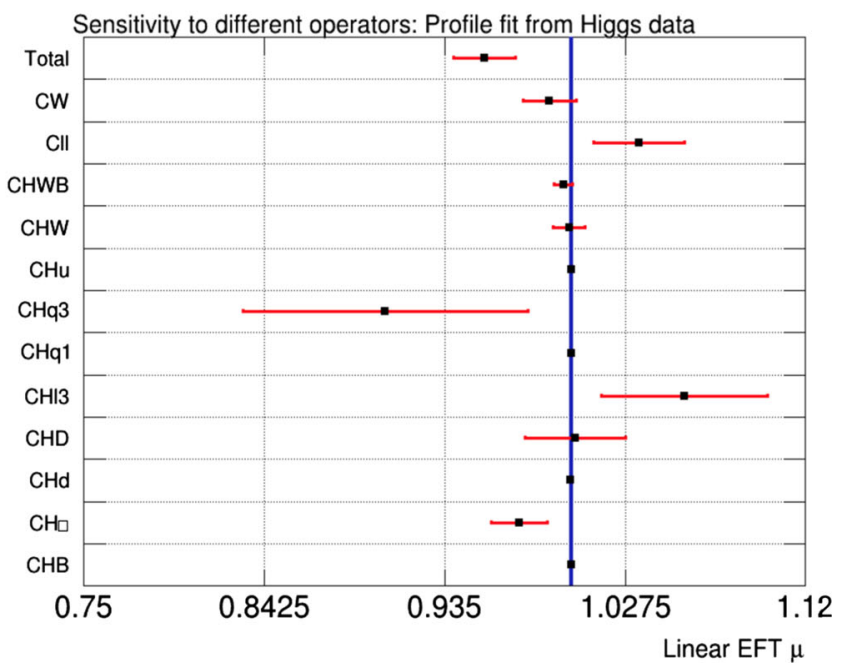

mentioned in the text. The entry "Total" accounts for the combination of the individual entries. In this figure as well as throughout this work we follow the operator nomenclature from Ref. [7] (Table 1)

\section{The Warsaw basis in VBS}

\subsection{EFT for the full process}

In this section, we investigate the effects of including all the Warsaw-basis operators on the computation of the VBS(ZZ) cross section. Some examples of the Feynman diagrams that contribute to the process are shown in Fig. 5.

In particular we found, numerically, the following expressions for the bosonic contribution to the total cross section:

$$
\begin{aligned}
& \frac{\sigma_{\mathrm{EFT}, \text { bosonic }}}{\sigma_{\mathrm{SM}}} \approx 1 .+0.047 \bar{c}_{\mathrm{HB}}-0.053 \bar{c}_{\mathrm{H}} \\
& \quad-0.0021 \bar{c}_{\widetilde{\mathrm{HB}}}+0.010 \bar{c}_{\mathrm{Hd}}-1.84 \bar{c}_{\mathrm{HD}} \\
& -3.86 \bar{c}_{\mathrm{Hl}}^{(3)}-0.017 \bar{c}_{\mathrm{Hq}}+5.61 \bar{c}_{\mathrm{Hq}^{(3)}} \\
& -0.033 \bar{c}_{\mathrm{Hu}}+0.59 \bar{c}_{\mathrm{HW}} \\
& -0.0041 \bar{c}_{\widetilde{\mathrm{HW}}}-0.69 \bar{c}_{\mathrm{HWB}}-0.022 \bar{c}_{\widetilde{\mathrm{HWB}}} \\
& +0.23 \bar{c}_{W}-0.086 \bar{c}_{\widetilde{W}} .
\end{aligned}
$$

For the fermionic contribution we have

$$
\begin{aligned}
& \frac{\sigma_{\mathrm{EFT}, \text { fermionic }}}{\sigma_{\mathrm{SM}}} \approx 1 .-3.23 \cdot 10^{-6} \bar{c}_{\mathrm{dd}} \\
& -2.89 \cdot 10^{-6} \bar{c}_{\mathrm{dd}}^{(1)}-3.86 \bar{c}_{\ell \ell}^{(1)}+0.0010 \bar{c}_{\mathrm{qd}}^{(1)} \\
& +1.80 \cdot 10^{-20} \bar{c}_{\mathrm{qd}}^{(8)}-1.93 \bar{c}_{\mathrm{qq}}^{(1)} \\
& -2.57 \bar{c}_{q q}^{(11)}-14.3 \bar{c}_{\mathrm{qq}}^{(3)}-10.3 \bar{c}_{\mathrm{qq}}^{(31)} \\
& -0.0049 \bar{c}_{q u}^{(1)}-2.51 \cdot 10^{-20} \bar{c}_{\mathrm{qu}}^{(8)}+0.00020 \bar{c}_{\mathrm{ud}}^{(1)} \\
& +1.62 \cdot 10^{-21} \bar{c}_{\mathrm{ud}}^{(8)}-0.0010 \bar{c}_{\mathrm{uu}}-0.00099 \bar{c}_{\mathrm{uu}}^{(1)} .
\end{aligned}
$$

Both of this expressions have been extracted from a simple numerical analysis of relatively small Monte Carlo samples 

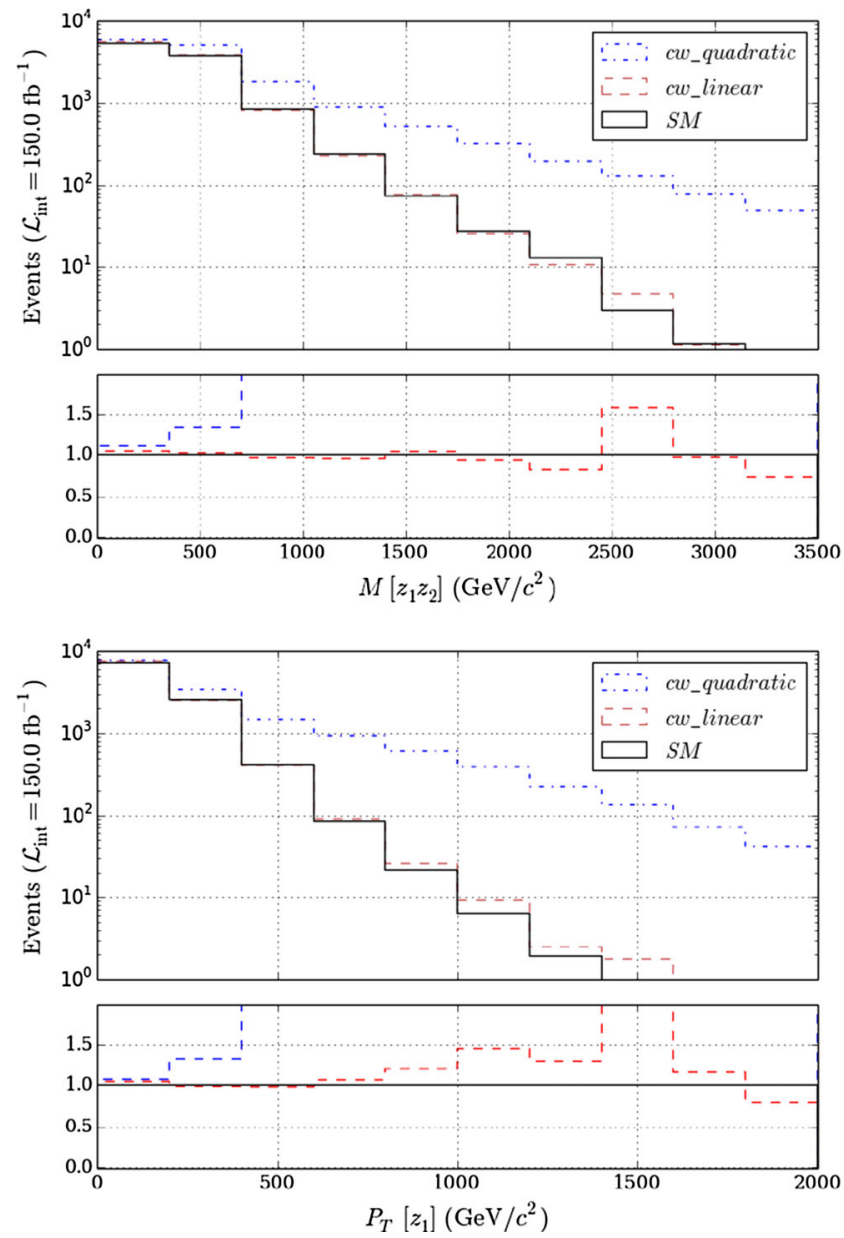

Fig. 4 Quadratic effects on some kinematic distributions. The red lines represent the linear contribution to the cross section ( $\mathrm{dim}=6$ interference with the SM) and the blue lines represent the previous contribution plus the purely $\operatorname{dim}=6$ term. We take the canonical values $\bar{c}_{W}=0.06$ and $\Lambda=1 \mathrm{TeV}$. The quadratic effects are relevant in general on the
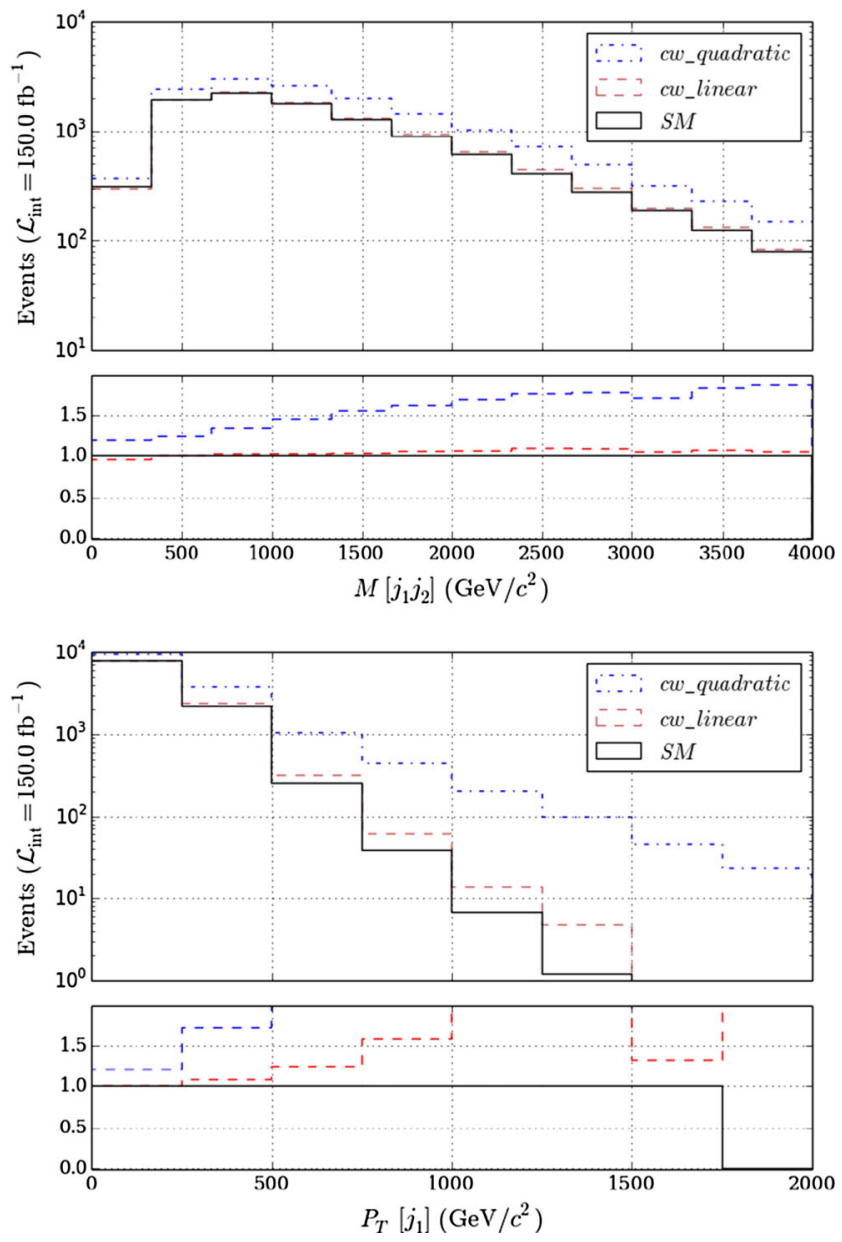

tails of the distributions, and in particular on the bins where the SM production vanishes. They also play a very important role in the bins where the interference with the SM is negative, since they may restore unitarity. $\Lambda=1 \mathrm{TeV}$ is the worst-case-scenario, for higher values of $\Lambda$ the difference between the linear and quadratic terms decreases
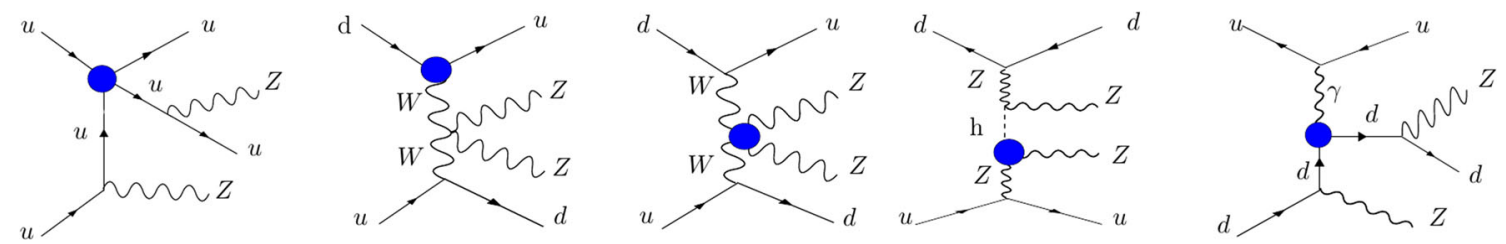

Fig. 5 Some of the Feynman diagrams contributing to the VBS(ZZ) process in dim $=6$ EFT. The blobs represent the dimension-six insertions

and hence are dominated by the MC uncertainty. Those we estimate to be of the order of $10 \%$ for each of the interference terms. The only purpose of displaying them here is to give an impression of the relative sensitivity of this process to the different EFT operators.

It is interesting to observe that the bosonic interference is generally positive, while the fermionic one is generally negative. This means that in the case that all the Wilson coefficients would have the same sign, both interferences could extensively cancel, giving rise to a very small SM deviation in the total cross section. For this reason, it is fundamental to define observables and regions where the EFT effects are maximised.

To understand the impact of the full $\operatorname{dim}=6$ basis, we defined different benchmark scenarios where we study the differential distributions for the most interesting VBS observables. 

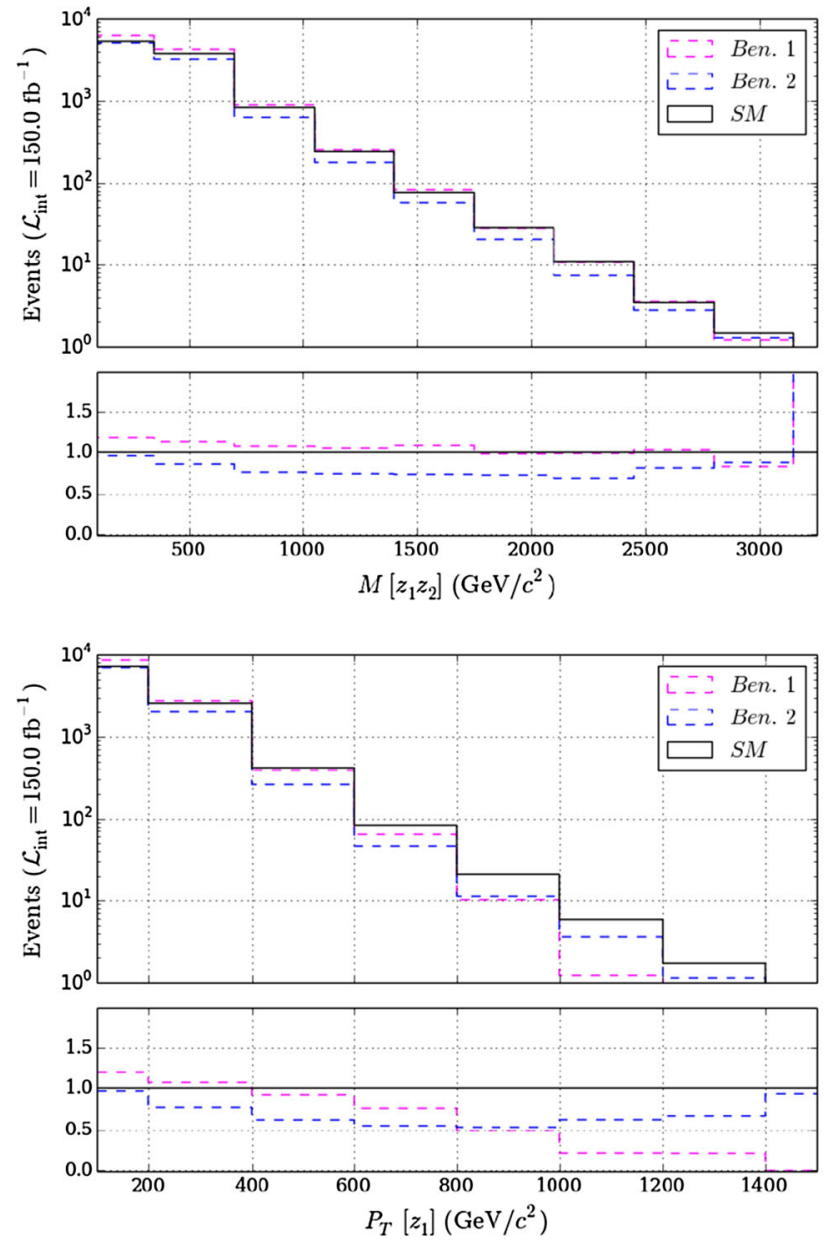

Fig. 6 Bosonic benchmarks B1 and B2. The "local" effects on individual observables and bins are very different from the global enhancement or decrease of the cross section. The observables related with transverse

Benchmarks 1 and 2 We consider all the bosonic, CP-even operators in the Warsaw basis, and, given the relative contribution of each of them to the total (linear) cross section, given by Eq. (5.1), we study two of the (infinite) possible solutions to the equation: one that gives an $\mathcal{O}(10 \%)$ enhancement and one that gives a $\mathcal{O}(10 \%)$ decrease (negative interference) to the SM total cross section. This is approximately the sensitivity we have to the process in LHC Run-2, and the order of the EW corrections to VBS at very high energies, see Refs. [66,127-129]. In Fig. 6 we see the effects of these two benchmarks for four different VBS observables, and in Table 1 we give the values used for the numerical simulation. For this part of the study we generated $9 \times 10^{5}$ events for each benchmark scenario, using the tools described in Sect. 4.

\subsection{Four-fermion operators}

In this section, we repeat the same procedure for purely fermionic operators. An interesting feature of four-fermion
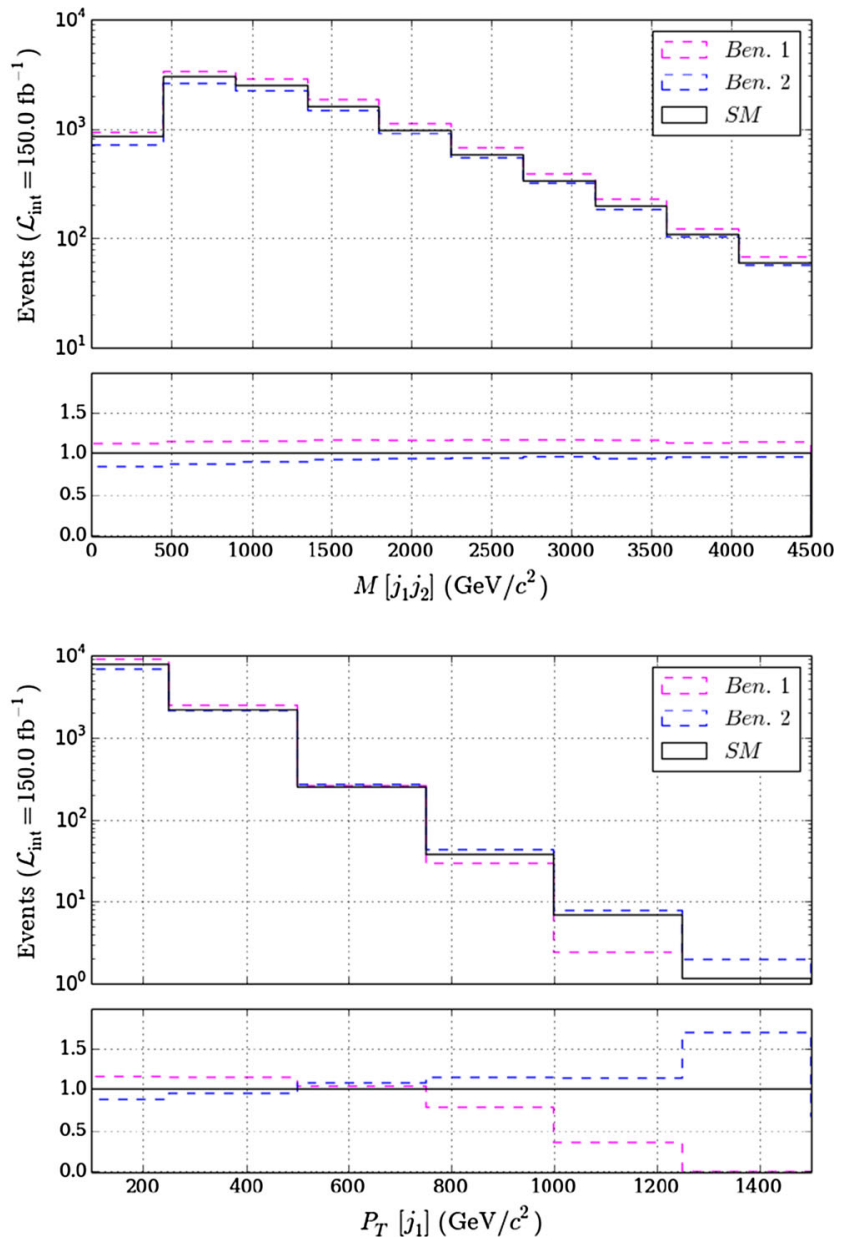

momentum seem to be more discriminating than the ones related to invariant masses. As anticipated, the EFT effects are larger on the tails of the distributions

Table 1 Benchmarks for bosonic operators. $\bar{c}_{i}=c_{i} \frac{v^{2}}{\Lambda^{2}}$. The number of significant digits used has been reduced in this table for the purpose of clarity. The CP-violating operators $\left\{\bar{c}_{\widetilde{\mathrm{W}}}, \bar{c}_{\widetilde{\mathrm{HW}}}, \bar{c}_{\widetilde{\mathrm{HWB}}}, \bar{c}_{\widetilde{\mathrm{HB}}}\right\}$ have been set to zero

\begin{tabular}{llc}
\hline Operator & Benchmark 1 & Benchmark 2 \\
\hline $\bar{c}_{\mathrm{HB}}$ & 0.0618 & -0.0157 \\
$\bar{c}_{H \square}$ & 0.0620 & 0.109 \\
$\bar{c}_{\mathrm{Hd}}$ & 0.0601 & 0.0872 \\
$\bar{c}_{\mathrm{HD}}$ & 0.0685 & 0.0409 \\
$\bar{c}_{\mathrm{Hl}}^{(3)}$ & 0.0761 & 0.0153 \\
$\bar{c}_{\mathrm{Hq}}^{(1)}$ & 0.0600 & -0.0248 \\
$\bar{c}_{\mathrm{Hq}}^{(3)}$ & 0.0391 & 0.0571 \\
$\bar{c}_{\mathrm{Hu}}$ & 0.0601 & -0.0481 \\
$\bar{c}_{\mathrm{HW}}$ & 0.0576 & -0.0360 \\
$\bar{c}_{\mathrm{HWB}}$ & 0.0628 & -0.0402 \\
$\bar{c}_{\mathrm{W}}$ & 0.0591 & -0.00507 \\
$\mu=\frac{\sigma_{\mathrm{EFT}}}{\sigma_{\mathrm{SM}}}$ & 0.89 & 1.14 \\
\hline
\end{tabular}


operators is that they are always generated at tree level in the UV-completion, whereas the rest of the dimension-six operators can be generated either at tree or loop level in the UV theory. This means that the effects of four-fermion operators will often be enhanced by a factor of $16 \pi^{2}$ with respect to the rest of the basis. This is particularly relevant for the case of the study here, since purely gauge operators, those built of three field strength tensors in the $\operatorname{dim}=6$ case of four field strength tensors in the dim $=8$ basis, are always generated from loops in the UV-completion, and hence always suppressed by $16 \pi^{2}$. For the original work on the PTG/LG classification see Refs. [45]. For more recent discussions see Refs. [11,130]

In this study, we consider all the fermionic operators. There are 12 operators that dominate the EFT contribution to this process, plus three additional ones which are very colour suppressed. Out of these, only $\mathcal{O}_{\ell \ell}$ can be constrained from the available fits, since it enters $G_{F}$ in the way described in Sect. 2. The rest of the four-fermion operators remain unconstrained, to the best of our knowledge.

Benchmarks 3, 4 and 5 As in the previous section, we find the solutions that enhance (B4) or diminish (B3) the SM cross section by approximately $10 \%$. In particular we chose the values given in Table 2. It is important to understand that these benchmarks are solutions to Eq. (5.2), which conspire to enhance or decrease the SM cross section, but the single entries of Table 2 have no physical meaning by themselves. Additionally we show the benchmark B5, which gives rise to the same total cross section as B3, but which has very different kinematics. This study can be seen in Figs. 7 and 8 .

\section{Di-jet observables}

Di-jet observables $\left(m_{j j}, \Delta \eta_{j j}\right)$ are characteristic quantities to any VBS study, since the main signature of such processes is given by their jets. Di-jet data in general represent a great opportunity to constrain four-quark operators at LHC, and some attempts in this direction have been presented in Refs. $[131,132]$. In this section we show the rapidity distributions corresponding to the different set-ups that were studied. This variable seems to be a very good pointer for NP effects in VBS.

\section{EFT in the background}

The main background at LHC for the previously studied process is the QCD induced process $p p \rightarrow z z j j$. This has a very large cross section compared with the VBS one and it is mainly discriminated from the signal thanks to the jet sig-
Table 2 Benchmarks for four-fermion operators. $\bar{c}_{i}=c_{i} \frac{v^{2}}{\Lambda^{2}}$. The number of significant digits used has been reduced in this table for the purpose of clarity. We have neglected the operators containing colour structures since they are very suppressed in this process, as shown in Eq. (5.2)

\begin{tabular}{lclc}
\hline Operator & Benchmark 3 & Benchmark 4 & Benchmark 5 \\
\hline $\bar{c}_{\mathrm{dd}}$ & -0.032 & 0.061 & 0.023 \\
$\bar{c}_{\mathrm{dd}}^{(1)}$ & 0.0077 & 0.061 & 0.092 \\
$\bar{c}_{\ell \ell}^{(1)}$ & -0.042 & 0.039 & 0.0036 \\
$\bar{c}_{\mathrm{qd}}^{(1)}$ & -0.033 & 0.060 & 0.076 \\
$\bar{c}_{\mathrm{qq}}^{(1)}$ & 0.0099 & 0.050 & -0.042 \\
$\bar{c}_{\mathrm{qq}}^{(11)}$ & 0.024 & 0.047 & -0.043 \\
$\bar{c}_{\mathrm{qq}}^{(3)}$ & 0.023 & -0.017 & -0.042 \\
$\bar{c}_{\mathrm{qq}}^{(31)}$ & -0.038 & 0.0031 & 0.094 \\
$\bar{c}_{\mathrm{qu}}^{(1)}$ & 0.051 & 0.060 & -0.0020 \\
$\bar{c}_{\mathrm{ud}}^{(1)}$ & 0.071 & 0.060 & -0.084 \\
$\bar{c}_{\mathrm{uu}}$ & -0.099 & 0.060 & -0.037 \\
$\bar{c}_{\mathrm{uu}}^{(1)}$ & -0.087 & 0.060 & -0.029 \\
$\mu=\frac{\sigma_{\mathrm{EFT}}}{\sigma_{\mathrm{SM}}}$ & 0.83 & 1.15 & 0.80 \\
\hline
\end{tabular}

nature of the latter. This background was analysed in LHC in Refs. [97, 133].

Moreover, in the available VBS(ZZ) analysis, the S/B discrimination was only achieved by means of a boosted decision tree (BDT) and a matrix element (ME) discriminator, described in Refs. [80,134].

Given this situation, it would not be sensible to add the EFT effects in the signal and not in the much larger background. In the following we show a preliminary study of the EFT effects in the background and we discuss which regions and observables are best for the observation of EFT effects (Fig. 9).

\subsection{Characterisation of the background}

For this study, we generated a sample of events of the QCD component, i.e. $\mathcal{O}\left(g_{s}^{2}\right)$, to the $p p \rightarrow Z Z j j$ process. The cross section for this component is much larger than the purely EW one, although this discrepancy can be minimised in the VBS fiducial region, yielding

$\mu_{\mathrm{S} / \mathrm{B}}=\left.\frac{\sigma_{\text {sig. }}}{\sigma_{\text {bkg. }}}\right|_{\mathrm{VBS} \text { region }} \approx 0.25$.

The sensitivity to the different $\operatorname{dim}=6$ operators can be extracted numerically in the same way as in Eqs. (5.1)-(5.2),

$$
\begin{aligned}
\frac{\sigma_{\mathrm{EFT}, \mathrm{bkg}}}{\sigma_{\mathrm{SM}, \mathrm{bkg}}} \approx & 1 .-0.00073 \bar{c}_{\mathrm{Hd}}-0.0036 \bar{c}_{\mathrm{HD}} \\
& +0.044 \bar{c}_{\mathrm{HG}}-0.00016 \bar{c}_{\widetilde{\mathrm{HG}}}
\end{aligned}
$$



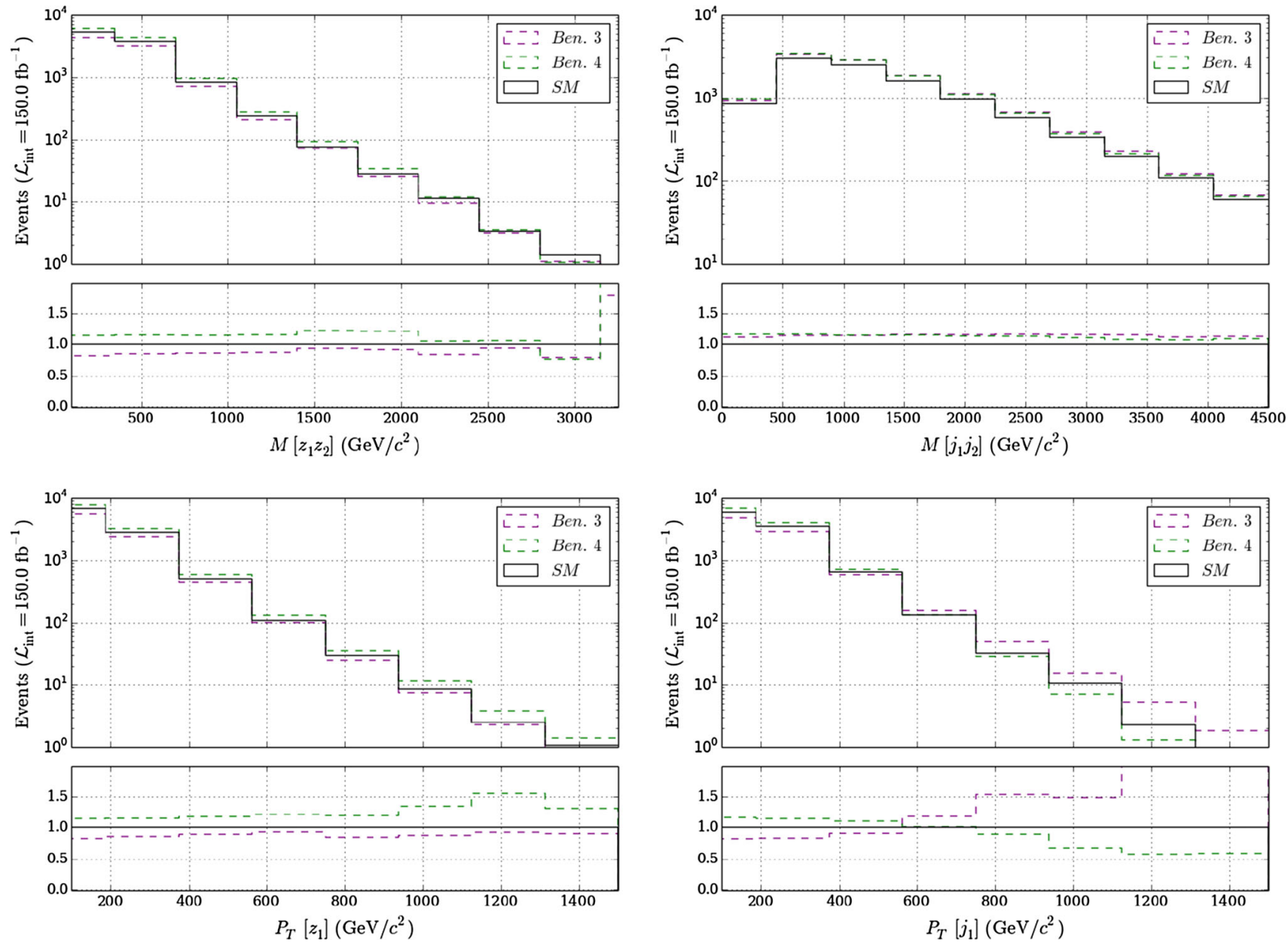

Fig. $7 \mathrm{SM}$ prediction and benchmark scenarios from Table 2. It is interesting to see that although one benchmark gives a total enhancement to the cross section and the other gives a decrease, in the tail of some distributions both seem to contribute positively
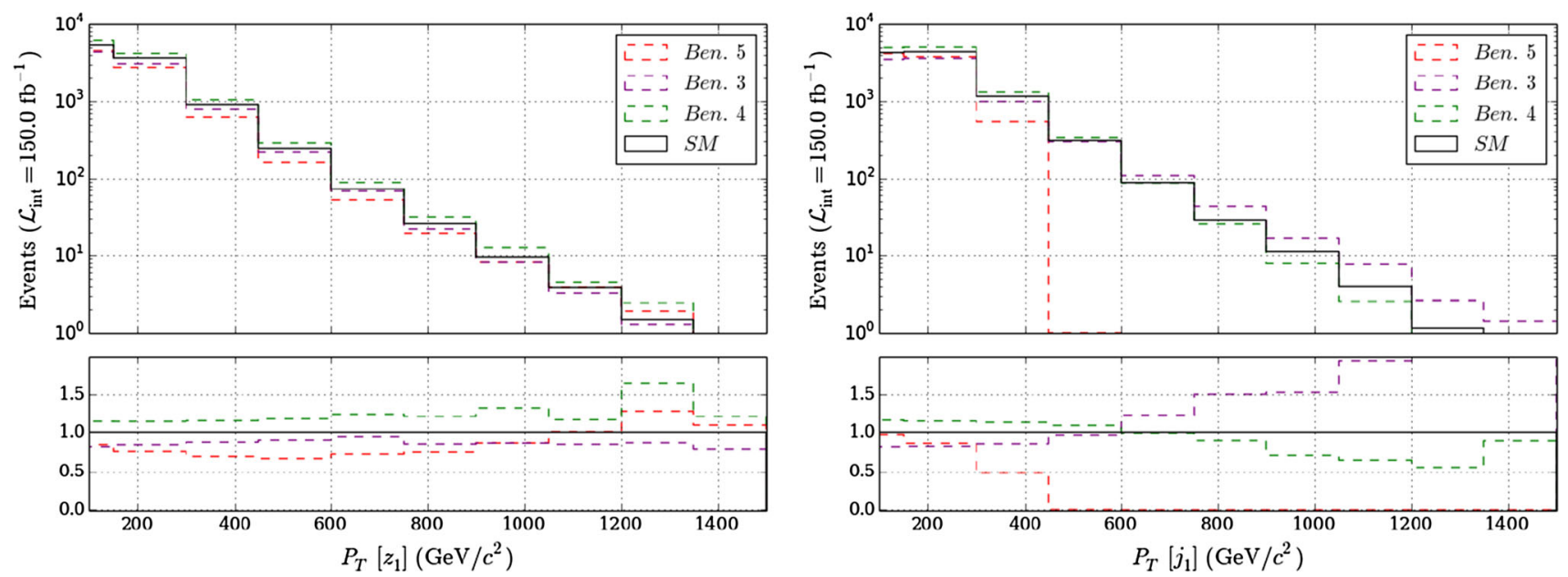

Fig. 8 Example of a benchmark scenario (B5 in Table 2) that is realistic in terms of cross section and $p_{\mathrm{T}}(Z)$, but has non-physical kinematics in the $p_{\mathrm{T}}(j)$ variable 

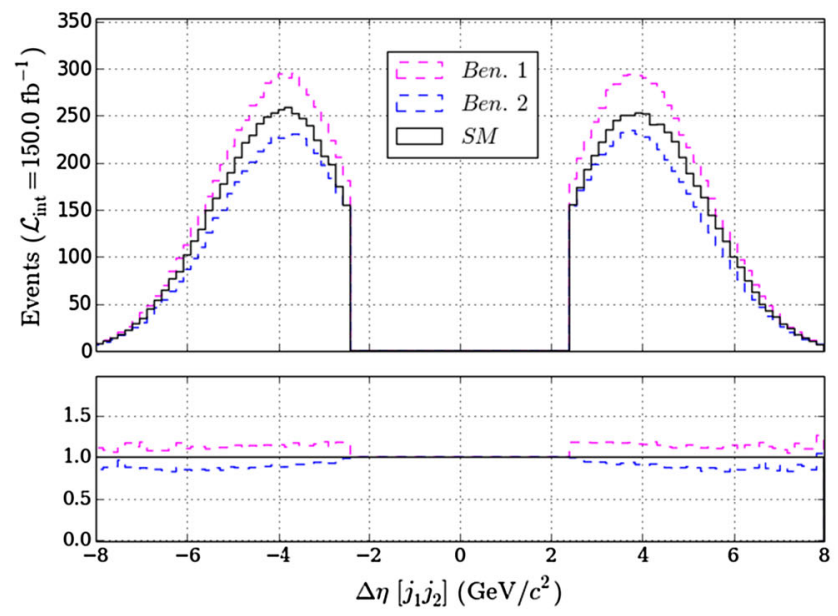
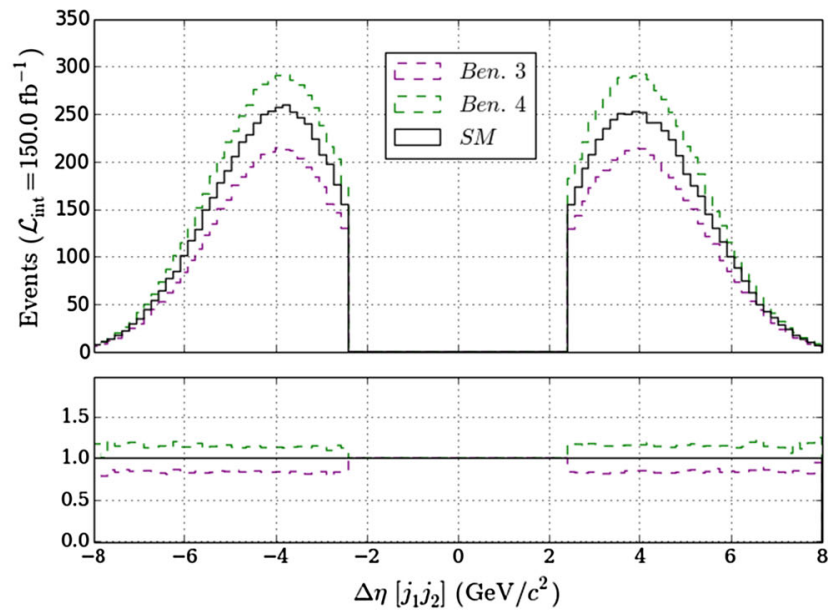
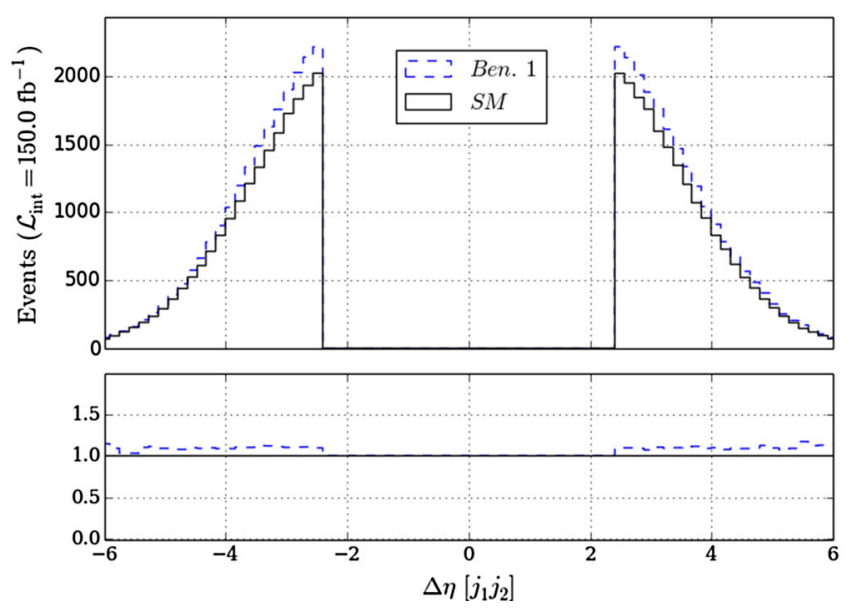

Fig. 9 Top: Bosonic benchmarks (left) and fermionic benchmarks (right). The $\Delta \eta$ variable seems to be a very good flag for new physics effects in VBS, where two rapidity peaks are always observed. Bottom: Effect of the B1 scenario in the background process, discussed in Sect.

$$
\begin{aligned}
& -0.077 \bar{c}_{\mathrm{Hl}}^{(3)}+0.018 \bar{c}_{\mathrm{Hq}}^{(1)}+0.17 \bar{c}_{\mathrm{Hq}}^{(3)} \\
& +0.0065 \bar{c}_{\mathrm{Hu}}+0.035 \bar{c}_{\mathrm{HWB}}-0.077 \bar{c}_{\ell \ell}^{(1)}
\end{aligned}
$$

This background is sensitive to fewer operators than the signal, and it is sensitive to other operators $\left\{\mathcal{O}_{\mathrm{HG}}, \mathcal{O}_{\widetilde{\mathrm{HG}}}\right\}$ to which the signal was blind. Some examples of Feynman diagrams for the dim $=6$ background can be seen in Fig. 10 . In order to see the effects of the dim $=6$ in the background, we generated a sample of background events, including the EFT operators corresponding to the benchmark scenario B1 in Table 1 , and leaving the new operators equal to zero. ${ }^{6}$ The first interesting observation is that this benchmark, which produced a negative interference of $20 \%$ in the signal cross section, gives rise to an enhancement in the background cross

\footnotetext{
$\overline{6 \text { The operator }} \mathcal{O}_{\mathrm{HG}}$ is very well understood through the gluon-gluon fusion process, and $\mathcal{O}_{\widetilde{\mathrm{HG}}}$ is $\mathrm{CP}$-odd.
}

7. The effects in the background are more subtle. Still it is important to notice that the number of background events is about one order of magnitude larger per bin, even in the VBS-enriched region studied in this work

section, of approximately $\approx 1 \%$. Given the fact that the background cross section is 4 times larger than the signal one, Eq. (7.1), a few percent of events in the background can be equivalent to an $\mathcal{O}(20 \%)$ number of signal events.

This means that the EFT effects in the background should always be well studied and understood. Else, an effect like this, background enhancement and signal reduction, could lead us to missing some interesting EFT effects if we only look at signals and total rates.

In Fig. 11 we show the corresponding plots for the background study.

\section{General strategy for EFT studies at LHC}

In this work we have shown that at least 11 bosonic and 12 fermionic dimension-six operators enter the VBS(ZZ) signal cross section. If we try to isolate the effects of each of 

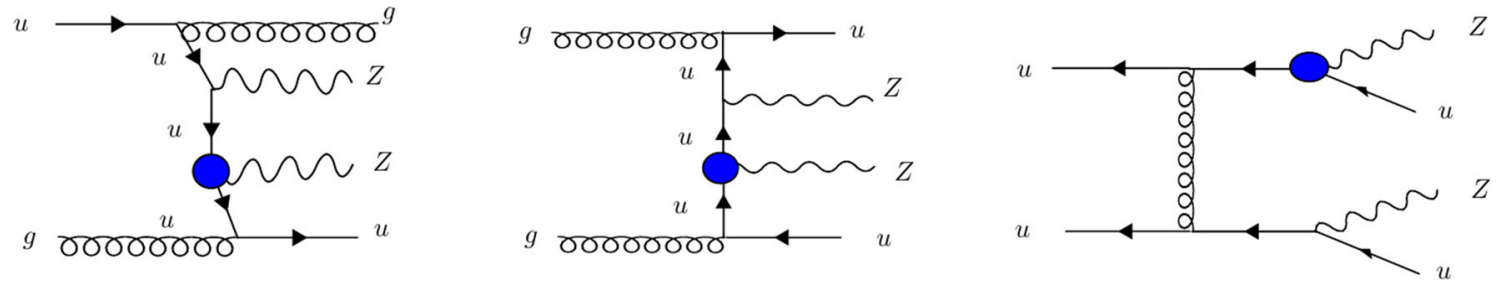

Fig. 10 Some of the Feynman diagrams contributing to the VBS(ZZ) dominant background process in dim $=6$ EFT. The blobs represent the dimension-six insertions
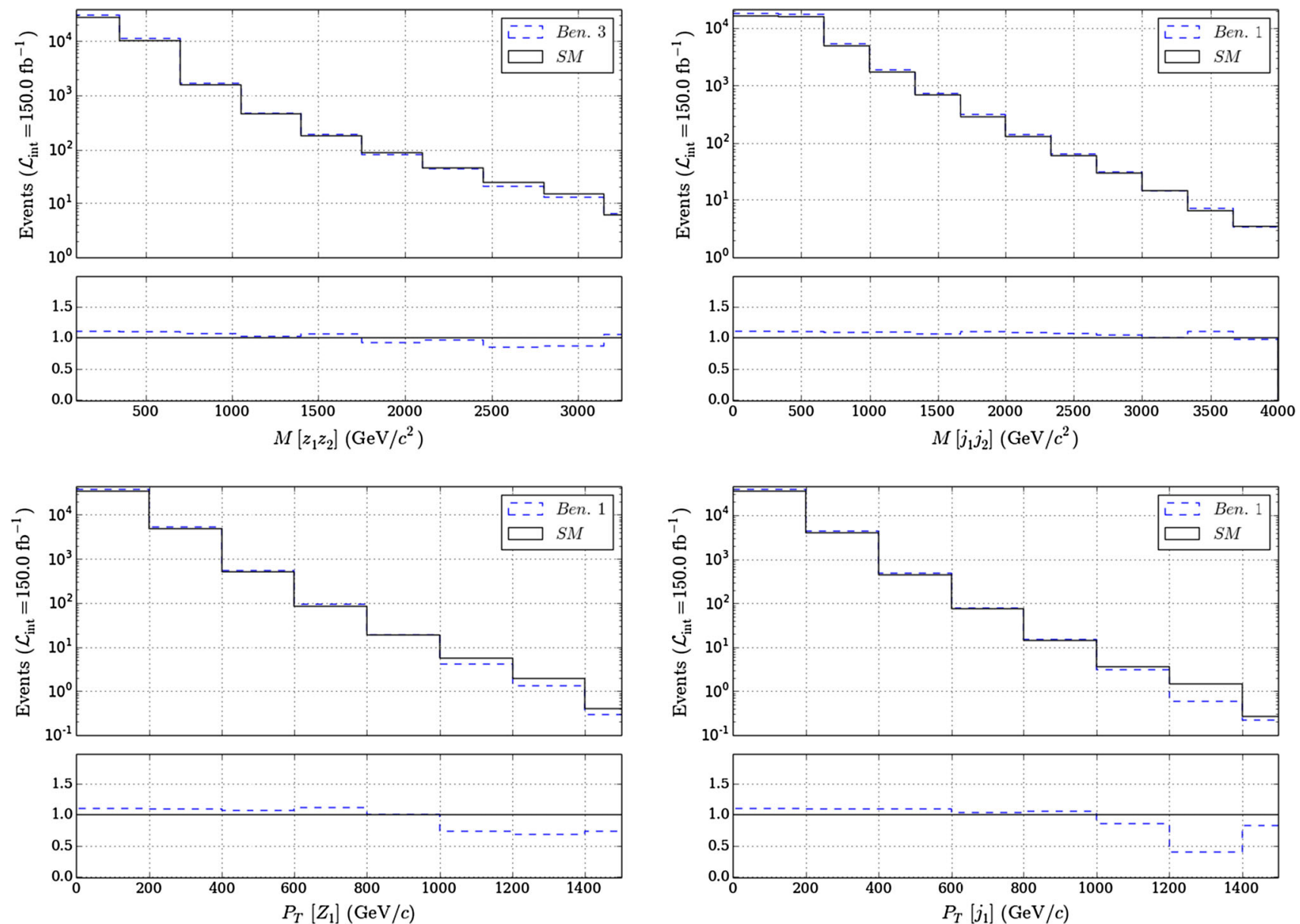

Fig. 11 Effect of the B1 scenario in the background process. The effect is in principle very small; however, it is important to remark that the number of background events is about one order of magnitude larger, per bin, than that for the signal

these operators using a single cross section measurement, the solutions to this system define a 22-dimensional manifold. In order to further constrain the space of solutions it is necessary to add further experimental measurements.

In this work we have proposed different VBS observables $^{7}$ : invariant mass of the two gauge bosons, invariant mass and rapidity of the di-jet system, transverse momentum of the leading gauge boson and jet and the rapidity sep-

\footnotetext{
7 We focus on the $\mathrm{ZZ}$ channel here, but this logic applies equally well to the other VBS channels.
}

aration between jets. As we have seen throughout Sect. 5, the EFT scales differently in different regions of phase space (with the high-energy "tails" being privileged). This means that a differential measurement with $n$ bins could be used to add $n$ equations to the aforementioned 23 variable system, reducing the number of directions in the solution hyperplane.

Moreover, if we assume factorisation of the EFT effects, by defining the amplitude as in Sect. 2.1, with one insertion per diagram and at tree level, the fiducial cross section can be written as

$\sigma_{\mathrm{EFT}}=\sigma_{\mathrm{SM}}+A \cdot\left(\mathcal{F}\left(\bar{c}_{1}, \bar{c}_{2}, \ldots, \bar{c}_{23}\right)\right)$, 
where $\mathcal{F}$ is a linear function of the Wilson coefficients $\bar{c}_{i}$, and $A$ is a global factor modulating the EFT contribution. In that case, the most important task is to find the relationship between the coefficients, $\mathcal{F}$, since the global factor $A$ can be constrained with the measurement of the fiducial cross section. The best way to do that includes using multivariate analysis (MVA) techniques, like the ones currently used in the experimental VBS searches, for example in the analysis discussed here, or in the analysis of the VBS(WZ) in ATLAS, in Ref. [135], where a 15 variable BDT was used.

It is always possible to add different measurements (from LEP, Higgs physics, etc.), in order to reduce the number of unknowns in the system of equations. But this should be dealt with taking great care: a bound set for a certain Wilson coefficient is only valid in the energy regime where the calculation is performed. In order to know the value of this coefficient at a different energy scale, the renormalisation group evolution of the coefficient has to be calculated. In that regard, it is safer to include more observables and more bins for signal and background measurements than to mix VBS-signal, Higgs-signal and LEP-signal measurements.

Ideally, gauge boson polarisation measurements, not studied in detail here, could also be useful. Such polarisation studies happen to be very interesting already at the SM level, as pointed out in recent work like Refs. [78, 136]. Furthermore, the study of the parton shower effects on the EFT distributions, might also shed new light on the problem. There are very few references in this direction so far, for example Ref. [123]. Last but not least, the study of the backgrounds, as shown in Sect. 7, is also necessary for a correct determination of the EFT effects.

\section{Conclusions}

At the current level of precision of LHC measurements, and the high level of agreement of these measurements with the SM predictions, it is advisable to perform any EFT analysis with a great deal of accuracy. For this purpose EFT operators cannot be studied on a case-by-case basis, and a global study of the set of dimension-six operators is necessary in the first place. In a second stage, dimension-eight as well as NLO and quadratic dimension-six effects need to be studied in order to improve the EFT theoretical uncertainties.

\footnotetext{
Acknowledgements We want to thank Giampiero Passarino for relevant comments on the manuscript, and Roberto Covarelli for relevant comments regarding the experimental set-up. We gratefully acknowledge Michael Trott for providing us with the likelihood functions for the fits presented in Refs. [117,118] as well as Ilaria Brivio for technical support with the SMEFTsim package, very interesting comments and discussions, and relevant input at the beginning of this project. We also want to thank Pietro Govoni for hosting us at the initial stage of this project, supported by a STSM Grant from COST Action CA16108, as well as the other members of the Action for interesting discussions.
}

Data Availability Statement This manuscript has no associated data or the data will not be deposited. [Authors' comment: This is a theoretical study and no experimental data has been listed.]

Open Access This article is distributed under the terms of the Creative Commons Attribution 4.0 International License (http://creativecomm ons.org/licenses/by/4.0/), which permits unrestricted use, distribution, and reproduction in any medium, provided you give appropriate credit to the original author(s) and the source, provide a link to the Creative Commons license, and indicate if changes were made. Funded by $\mathrm{SCOAP}^{3}$.

\section{References}

1. R. Contino, M. Ghezzi, C. Grojean, M. Muhlleitner, M. Spira, Effective Lagrangian for a light Higgs-like scalar. JHEP 07, 035 (2013). arXiv:1303.3876 [hep-ph]

2. A. Azatov, C. Grojean, A. Paul, E. Salvioni, Taming the offshell Higgs boson. Zh. Eksp. Teor. Fiz. 147, 410-425 (2015). arXiv:1406.6338 [hep-ph]

3. A. Azatov, C. Grojean, A. Paul, E. Salvioni, Taming the off-shell Higgs boson. J. Exp. Theor. Phys. 120, 354 (2015)

4. R. Contino, M. Ghezzi, C. Grojean, M. Mühlleitner, M. Spira, eHDECAY: an implementation of the Higgs effective Lagrangian into HDECAY. Comput. Phys. Commun. 185, 3412-3423 (2014). arXiv:1403.3381 [hep-ph]

5. L. Berthier, M. Trott, Towards consistent electroweak precision data constraints in the SMEFT. JHEP 05, 024 (2015). arXiv: 1502.02570 [hep-ph]

6. M. Trott, On the consistent use of constructed observables. JHEP 02, 046 (2015). arXiv:1409.7605 [hep-ph]

7. R. Alonso, E.E. Jenkins, A.V. Manohar, M. Trott, Renormalization group evolution of the standard model dimension six operators III: gauge coupling dependence and phenomenology. JHEP 04, 159 (2014). arXiv:1312.2014 [hep-ph]

8. E.E. Jenkins, A.V. Manohar, M. Trott, Renormalization group evolution of the standard model dimension six operators II: Yukawa dependence. JHEP 01, 035 (2014). arXiv:1310.4838 [hep-ph]

9. E.E. Jenkins, A.V. Manohar, M. Trott, Naive dimensional analysis counting of gauge theory amplitudes and anomalous dimensions. Phys. Lett. B 726, 697-702 (2013). arXiv:1309.0819 [hep-ph]

10. E.E. Jenkins, A.V. Manohar, M. Trott, Renormalization group evolution of the standard model dimension six operators I: formalism and lambda dependence. JHEP 10, 087 (2013). arXiv:1308.2627 [hep-ph]

11. E.E. Jenkins, A.V. Manohar, M. Trott, On gauge invariance and minimal coupling. JHEP 09, 063 (2013). arXiv:1305.0017 [hep$\mathrm{ph}]$

12. P. Artoisenet et al., A framework for Higgs characterisation. JHEP 11, 043 (2013). arXiv:1306.6464 [hep-ph]

13. A. Alloul, B. Fuks, V. Sanz, Phenomenology of the Higgs effective lagrangian via Feynrules. JHEP 04, 110 (2014). arXiv: 1310.5150 [hep-ph]

14. J. Ellis, V. Sanz, T. You, Complete Higgs sector constraints on dimension-6 operators. JHEP 07, 036 (2014). arXiv:1404.3667 [hep-ph]

15. A. Falkowski, F. Riva, Model-independent precision constraints on dimension-6 operators. JHEP 02, 039 (2015). arXiv:1411.0669 [hep-ph]

16. I. Low, R. Rattazzi, A. Vichi, Theoretical constraints on the Higgs effective couplings. JHEP 04, 126 (2010). arXiv:0907.5413 [hep$\mathrm{ph}]$

17. C. Degrande, N. Greiner, W. Kilian, O. Mattelaer, H. Mebane, T. Stelzer, S. Willenbrock, C. Zhang, Effective field theory: a modern 
approach to anomalous couplings. Ann. Phys. 335, 21-32 (2013). arXiv: 1205.4231 [hep-ph]

18. C.-Y. Chen, S. Dawson, C. Zhang, Electroweak effective operators and Higgs physics. Phys. Rev. D 89(1), 015016 (2014). arXiv: 1311.3107 [hep-ph]

19. R. Grober, M. Muhlleitner, M. Spira, J. Streicher, NLO QCD corrections to Higgs pair production including dimension-6 operators. JHEP 09, 092 (2015). arXiv:1504.06577 [hep-ph]

20. C. Englert, M. McCullough, M. Spannowsky, Combining LEP and LHC to bound the Higgs width. Nucl. Phys. B 902, 440-457 (2016). arXiv:1504.02458 [hep-ph]

21. C. Englert, I. Low, M. Spannowsky, On-shell interference effects in Higgs boson final states. Phys. Rev. D 91(7), 074029 (2015). arXiv:1502.04678 [hep-ph]

22. C. Englert, A. Freitas, M.M. Mühlleitner, T. Plehn, M. Rauch, M. Spira, K. Walz, Precision measurements of Higgs couplings: implications for new physics scales. J. Phys. G41, 113001 (2014). arXiv:1403.7191 [hep-ph]

23. A. Biekötter, A. Knochel, M. Krämer, D. Liu, F. Riva, Vices and virtues of Higgs effective field theories at large energy. Phys. Rev. D 91, 055029 (2015). arXiv: 1406.7320 [hep-ph]

24. R.S. Gupta, A. Pomarol, F. Riva, BSM primary effects. Phys. Rev. D 91(3), 035001 (2015). arXiv:1405.0181 [hep-ph]

25. J. Elias-Miro, J.R. Espinosa, E. Masso, A. Pomarol, Higgs windows to new physics through $\mathrm{d}=6$ operators: constraints and one-loop anomalous dimensions. JHEP 11, 066 (2013). arXiv:1308.1879 [hep-ph]

26. J. Elias-Miro, J.R. Espinosa, E. Masso, A. Pomarol, Renormalization of dimension-six operators relevant for the Higgs decays $h \rightarrow \gamma \gamma, \gamma Z$. JHEP 08, 033 (2013). arXiv:1302.5661 [hep-ph]

27. A. Pomarol, F. Riva, Towards the ultimate SM Fit to close in on Higgs physics. JHEP 01, 151 (2014). arXiv:1308.2803 [hep-ph]

28. E. Masso, An effective guide to beyond the standard model physics. JHEP 10, 128 (2014). arXiv:1406.6376 [hep-ph]

29. J. de Blas, M. Chala, M. Perez-Victoria, J. Santiago, Observable effects of general new scalar particles. JHEP 04, 078 (2015). arXiv: 1412.8480 [hep-ph]

30. T. Corbett, O.J.P. Eboli, D. Goncalves, J. Gonzalez-Fraile, T. Plehn, M. Rauch, The non-linear Higgs legacy of the LHC run I. (2015) arXiv:1511.08188 [hep-ph]

31. T. Corbett, O.J.P. Eboli, D. Goncalves, J. Gonzalez-Fraile, T. Plehn, M. Rauch, The Higgs legacy of the LHC run I. JHEP 08, 156 (2015). arXiv:1505.05516 [hep-ph]

32. R. Contino, A. Falkowski, F. Goertz, C. Grojean, F. Riva, On the validity of the effective field theory approach to SM precision tests. JHEP 07, 144 (2016). arXiv:1604.06444 [hep-ph]

33. D. Barducci et al., Interpreting top-quark LHC measurements in the standard-model effective field theory. (2018) arXiv:1802.07237 [hep-ph]

34. M. Chala, G. Durieux, C. Grojean, L. de Lima, O. Matsedonskyi, Minimally extended SILH. JHEP 06, 088 (2017). arXiv:1703.10624 [hep-ph]

35. J. de Blas, J.C. Criado, M. Perez-Victoria, J. Santiago, Effective description of general extensions of the standard model: the complete tree-level dictionary. JHEP 03, 109 (2018). arXiv:1711.10391 [hep-ph]

36. J. de Blas, O. Eberhardt, C. Krause, Current and future constraints on Higgs couplings in the nonlinear effective theory. JHEP 07, 048 (2018). arXiv:1803.00939 [hep-ph]

37. B. Henning, X. Lu, H. Murayama, How to use the standard model effective field theory. JHEP 01, 023 (2016). arXiv:1412.1837 [hep-ph]

38. M. Boggia, R. Gomez-Ambrosio, G. Passarino, Low energy behaviour of standard model extensions. JHEP 05, 162 (2016). arXiv:1603.03660 [hep-ph]
39. F. del Aguila, Z. Kunszt, J. Santiago, One-loop effective lagrangians after matching. Eur. Phys. J. C 76(5), 244 (2016). arXiv:1602.00126 [hep-ph]

40. T. Appelquist, J. Carazzone, Infrared singularities and massive fields. Phys. Rev. D 11, 2856 (1975)

41. C. Arzt, Reduced effective Lagrangians. Phys. Lett. B 342, 189195 (1995). arXiv:hep-ph/9304230 [hep-ph]

42. C. Arzt, M.B. Einhorn, J. Wudka, Patterns of deviation from the standard model. Nucl. Phys. B 433, 41-66 (1995). arXiv:hep-ph/9405214 [hep-ph]

43. H. Georgi, Effective field theory. Ann. Rev. Nucl. Part. Sci. 43, 209-252 (1993)

44. M.S. Chanowitz, M. Golden, H. Georgi, Low-energy theorems for strongly interacting W's and Z's. Phys. Rev. D 36, 1490 (1987)

45. M.B. Einhorn, J. Wudka, The bases of effective field theories. Nucl. Phys. B 876, 556-574 (2013). arXiv:1307.0478 [hep-ph]

46. I. Brivio, M. Trott, The standard model as an effective field theory (2017). arXiv:1706.08945 [hep-ph]

47. M. Boggia et al., The HiggsTools handbook: a beginners guide to decoding the Higgs sector. J. Phys. G45(6), 065004 (2018). arXiv:1711.09875 [hep-ph]

48. A.V. Manohar, Introduction to effective field theories, in Les Houches summer school: EFT in Particle Physics and Cosmology Les Houches, Chamonix Valley, France, July 3-28, 2017 (2018). arXiv:1804.05863 [hep-ph]

49. P.W. Higgs, Broken symmetries and the masses of gauge bosons. Phys. Rev. Lett. 13, 508-509 (1964)

50. F. Englert, R. Brout, Broken symmetry and the mass of gauge vector mesons. Phys. Rev. Lett. 13, 321-323 (1964)

51. G.S. Guralnik, C.R. Hagen, T.W.B. Kibble, Global conservation laws and massless particles. Phys. Rev. Lett. 13, 585-587 (1964)

52. P.W. Higgs, Spontaneous symmetry breakdown without massless bosons. Phys. Rev. 145, 1156-1163 (1966)

53. T.W.B. Kibble, Symmetry breaking in nonAbelian gauge theories. Phys. Rev. 155, 1554-1561 (1967)

54. S. Weinberg, Baryon and Lepton nonconserving processes. Phys. Rev. Lett. 43, 1566-1570 (1979)

55. B. Grzadkowski, M. Iskrzynski, M. Misiak, J. Rosiek, Dimensionsix terms in the standard model lagrangian. JHEP 10, 085 (2010). arXiv:1008.4884 [hep-ph]

56. B. Henning, X. Lu, T. Melia, H. Murayama, 2, 84, 30, 993, 560, $15456,11962,261485, .:$ higher dimension operators in the SM EFT. JHEP 08, 016 (2017). arXiv:1512.03433 [hep-ph]

57. O.J.P. Eboli, M.C. Gonzalez-Garcia, J.K. Mizukoshi, p p $\rightarrow$ j j e+- mu+- nu nu and j j e+- mu-+ nu nu at $\mathrm{O}($ alpha(em)**6) and $\mathrm{O}($ alpha $(\mathrm{em}) * * 4$ alpha(s)**2) for the study of the quartic electroweak gauge boson vertex at CERN LHC. Phys. Rev. D 74, 073005 (2006). arXiv:hep-ph/0606118 [hep-ph]

58. O.J.P. Eboli, M.C. Gonzalez-Garcia, Classifying the bosonic quartic couplings. Phys. Rev. D 93(9), 093013 (2016). arXiv:1604.03555 [hep-ph]

59. K. Hagiwara, S. Ishihara, R. Szalapski, D. Zeppenfeld, Lowenergy effects of new interactions in the electroweak boson sector. Phys. Rev. D 48, 2182-2203 (1993)

60. M. Ghezzi, R. Gomez-Ambrosio, G. Passarino, S. Uccirati, NLO Higgs effective field theory and $\kappa$-framework. JHEP 07, 175 (2015). arXiv:1505.03706 [hep-ph]

61. A. Helset, M. Paraskevas, M. Trott, Gauge fixing the standard model effective field theory. Phys. Rev. Lett. 120(25), 251801 (2018). arXiv:1803.08001 [hep-ph]

62. A. Dedes, W. Materkowska, M. Paraskevas, J. Rosiek, K. Suxho, Feynman rules for the standard model effective field theory in $\mathrm{R}_{x i}$ -gauges. JHEP 06, 143 (2017). arXiv:1704.03888 [hep-ph]

63. S. Dawson, P.P. Giardino, Higgs decays to $Z Z$ and $Z \gamma$ in the standard model effective field theory: an NLO analysis. Phys. Rev. D 97(9), 093003 (2018). arXiv:1801.01136 [hep-ph] 
64. E. Accomando, A. Ballestrero, A. Belhouari, E. Maina, Isolating vector boson scattering at the LHC: gauge cancellations and the equivalent vector boson approximation vs complete calculations. Phys. Rev. D 74, 073010 (2006). arXiv:hep-ph/0608019 [hep-ph]

65. M. Rauch, Vector-Boson fusion and Vector-Boson scattering (2016). arXiv: 1610.08420 [hep-ph]

66. B. Biedermann, A. Denner, M. Pellen, Large electroweak corrections to vector-boson scattering at the Large Hadron Collider. Phys. Rev. Lett. 118(26), 261801 (2017). arXiv:1611.02951 [hep$\mathrm{ph}]$

67. U. Baur, E.W.N. Glover, Z Boson pair production via vector boson scattering and the search for the Higgs Boson at Hadron supercolliders. Nucl. Phys. B 347, 12-66 (1990)

68. A. Ballestrero, D.Buarque Franzosi, L. Oggero, E. Maina, Vector Boson scattering at the LHC: counting experiments for unitarized models in a full six fermion approach. JHEP 03, 031 (2012). arXiv:1112.1171 [hep-ph]

69. R. Kleiss, W.J. Stirling, Anomalous High-energy behavior in Boson fusion. Phys. Lett. B 182, 75 (1986)

70. K. Philippides, W.J. Stirling, Restoring good high-energy behavior in Higgs production via W fusion at the LHC. Eur. Phys. J. C 9, 181-195 (1999). arXiv:hep-ph/9901451 [hep-ph]

71. A. Ballestrero, D Buarque Franzosi, E. Maina, Exploring alternative symmetry breaking mechanisms at the LHC with 7, 8 and 10 TeV total energy. JHEP 05, 083 (2012). arXiv: 1203.2771 [hep-ph]

72. W. Kilian, T. Ohl, J. Reuter, M. Sekulla, High-energy vector boson scattering after the Higgs discovery. Phys. Rev. D 91, 096007 (2015). arXiv:1408.6207 [hep-ph]

73. R.L. Delgado, A. Dobado, F.J. Llanes-Estrada, Unitarity, analyticity, dispersion relations, and resonances in strongly interacting $W_{L} W_{L}, Z_{L} Z_{L}$, and hh scattering. Phys. Rev. D 91(7), 075017 (2015). arXiv:1502.04841 [hep-ph]

74. A. Dobado, F.-K. Guo, F.J. Llanes-Estrada, Production cross section estimates for strongly-interacting electroweak symmetry breaking sector resonances at particle colliders. Commun. Theor. Phys. 64, 701-709 (2015). arXiv:1508.03544 [hep-ph]

75. M. Sekulla, W. Kilian, T. Ohl, J. Reuter, Effective field theory and unitarity in vector boson scattering. PoS LHCP 2016, 052 (2016). arXiv:1610.04131 [hep-ph]

76. R. Gomez-Ambrosio, Study of VBF/VBS in the LHC at $13 \mathrm{TeV}$, the EFT approach, in 28th Rencontres de Blois on Particle Physics and Cosmology Blois, France, May 29-June 3, 2016. (2016). arXiv:1610.07491 [hep-ph]

77. D Buarque Franzosi, Implications of vector boson scattering unitarity in composite Higgs models. PoS EPS-HEP 2017, 264 (2017)

78. S. Brass, C. Fleper, W. Kilian, J. Reuter, M. Sekulla, Transversal modes and Higgs bosons in electroweak vector-Boson scattering at the LHC (2018). arXiv:1807.02512 [hep-ph]

79. R. L. Delgado, A. Dobado, F. J. Llanes-Estrada, Unitarized HEFT for strongly interacting longitudinal electroweak gauge bosons with resonances, in 6th Large Hadron Collider Physics Conference (LHCP 2018) Bologna, Italy, June 4-9, 2018. (2018). arXiv:1808.04413 [hep-ph]

80. CMS Collaboration, A. M. Sirunyan et al., Measurement of vector boson scattering and constraints on anomalous quartic couplings from events with four leptons and two jets in proton-proton collisions at sqrt(s) $=13 \mathrm{TeV}$, arXiv: 1708.02812 [hep-ex]

81. K.J.F. Gaemers, G.J. Gounaris, Polarization amplitudes for e+ e- $\rightarrow$ W+ W- and e+ e- $\rightarrow$ Z Z. Z. Phys. C 1, 259 (1979)

82. A. Dobado, D. Espriu, M.J. Herrero, Chiral Lagrangians as a tool to probe the symmetry breaking sector of the SM at LEP. Phys. Lett. B 255, 405-414 (1991)

83. B. Grinstein, M.B. Wise, Operator analysis for precision electroweak physics. Phys. Lett. B 265, 326-334 (1991)
84. K. Hagiwara, S. Ishihara, R. Szalapski, D. Zeppenfeld, Lowenergy constraints on electroweak three gauge boson couplings. Phys. Lett. B 283, 353-359 (1992)

85. J. Wudka, Electroweak effective Lagrangians. Int. J. Mod. Phys. A 9, 2301-2362 (1994). arXiv:hep-ph/9406205 [hep-ph]

86. M. Fabbrichesi, M. Pinamonti, A. Tonero, A. Urbano, Vector boson scattering at the LHC: a study of the WW $\rightarrow$ WW channels with the Warsaw cut. Phys. Rev. D 93(1), 015004 (2016). arXiv:1509.06378 [hep-ph]

87. A. Falkowski, M. Gonzalez-Alonso, A. Greljo, D. Marzocca, M. Son, Anomalous triple gauge couplings in the effective field theory approach at the LHC. JHEP 02, 115 (2017). arXiv: 1609.06312 [hep-ph]

88. T. Corbett, O.J.P. Éboli, M.C. Gonzalez-Garcia, Unitarity constraints on dimension-six operators. Phys. Rev. D 91(3), 035014 (2015). arXiv:1411.5026 [hep-ph]

89. T. Corbett, M.J. Dolan, C. Englert, K. Nordström, Anomalous neutral gauge boson interactions and simplified models. Phys. Rev. D 97(11), 115040 (2018). arXiv: 1710.07530 [hep-ph]

90. H. Mebane, N. Greiner, C. Zhang, S. Willenbrock, Constraints on electroweak effective operators at one loop. Phys. Rev. D 88(1), 015028 (2013). arXiv:1306.3380 [hep-ph]

91. R. Rahaman, R.K. Singh, Constraining anomalous gauge boson couplings in $e^{+} e^{-} \rightarrow W^{+} W^{-}$using polarization asymmetries with polarized beams (2017). arXiv:1711.04551 [hep-ph]

92. DELPHI, OPAL, ALEPH, L3 Collaboration, J. Jousset, Triple Couplings Of Electroweak Gauge Bosons At LEP2, in Proceedings, 33rd Rencontres de Moriond 98 electrowek interactions and unified theories: Les Arcs, France, Mar 14-21, 1998, pp. 77-82, Edition Frontieres. Edition Frontieres, Paris, (1998)

93. DELPHI, OPAL, ALEPH, L3 Collaboration, B. de la Cruz, Production of $\mathrm{Z}$ pairs at LEP2 and neutral anomalous couplings, in Results and perspectives in particle physics. Proceedings, Les Rencontres de Physique de la Vallee d'Aoste, La Thuile, Italy, 2002, pp. 681-691 (2002)

94. ALEPH Collaboration, S. Schael et al., Improved measurement of the triple gauge-boson couplings gamma W W and Z W W in e+ e- collisions, Phys. Lett. B 614, 7-26 (2005)

95. DELPHI Collaboration, J. Abdallah et al., Study of W boson polarisations and Triple Gauge boson Couplings in the reaction e+e- $\longrightarrow$ W+W- at LEP 2, Eur. Phys. J. C54, 345-364 (2008). arXiv:0801.1235 [hep-ex]

96. ALEPH Collaboration, S. Schael et al., Measurement of Z-pair production in $\mathrm{e}+\mathrm{e}$ - collisions and constraints on anomalous neutral gauge couplings, JHEP 04, 124 (2009)

97. ATLAS Collaboration, M. Aaboud et al., $Z Z \rightarrow \ell^{+} \ell^{-} \ell^{\prime+} \ell^{\prime-}$ cross-section measurements and search for anomalous triple gauge couplings in $13 \mathrm{TeV} p p$ collisions with the ATLAS detector, Phys. Rev. D 97(3), 032005 (2018). arXiv:1709.07703 [hep-ex]

98. ATLAS Collaboration, T. A. collaboration, $Z Z \rightarrow \ell^{+} \ell^{-} \ell^{\prime+} \ell^{\prime-}$ cross-section measurements and aTGC search in $13 \mathrm{TeV} p p$ collisions with the ATLAS detector,

99. C.M.S. Collaboration, A.M. Sirunyan et al., Measurements of the $\mathrm{pp} \rightarrow \mathrm{ZZ}$ production cross section and the $\mathrm{Z} \rightarrow 4 \ell$ branching fraction, and constraints on anomalous triple gauge couplings at $\sqrt{s}=13$ TeV. Eur. Phys. J. C 78, 165 (2018). arXiv:1709.08601 [hep-ex]. [Erratum: Eur. Phys. J. C78, no.6,515(2018)]

100. ALEPH Collaboration, A. Collaboration, Constraints on anomalous quartic gauge boson couplings from photon pair events from $189 \mathrm{GeV}$ to $209 \mathrm{GeV}$, in Lepton and photon interactions at high energies. In: Proceedings, 20th International Symposium, LP 2001, Rome, Italy, July 23-28, 2001 (2001)

101. O.P.A.L. Collaboration, G. Abbiendi et al., Constraints on anomalous quartic gauge boson couplings from nu anti-nu gamma gamma and q anti-q gamma gamma events at LEP-2. Phys. Rev. D 70, 032005 (2004). arXiv:hep-ex/0402021 [hep-ex] 
102. M. Bordone, A. Greljo, G. Isidori, D. Marzocca, A. Pattori, Higgs Pseudo observables and radiative corrections. Eur. Phys. J. C 75(8), 385 (2015). arXiv:1507.02555 [hep-ph]

103. A. David, G. Passarino, Through precision straits to next standard model heights. Rev. Phys. 1, 13-28 (2016). arXiv:1510.00414 [hep-ph]

104. A. Greljo, G. Isidori, J.M. Lindert, D. Marzocca, Pseudoobservables in electroweak Higgs production. Eur. Phys. J. C 76(3), 158 (2016). arXiv:1512.06135 [hep-ph]

105. I. Brivio, Y. Jiang, M. Trott, The SMEFTsim package, theory and tools. JHEP 12, 070 (2017). arXiv:1709.06492 [hep-ph]

106. J. S. Gainer et al., Adding pseudo-observables to the four-Lepton experimentalist's toolbox (2018) arXiv:1808.00965 [hep-ph]

107. LHC Higgs Cross Section Working Group Collaboration, D. de Florian et al., Handbook of LHC Higgs Cross Sections: 4. Deciphering the Nature of the Higgs Sector (2016) arXiv:1610.07922 [hep-ph]

108. C. Degrande, A basis of dimension-eight operators for anomalous neutral triple gauge boson interactions. JHEP 02, 101 (2014). arXiv:1308.6323 [hep-ph]

109. B. Bellazzini, F. Riva, $Z Z$ and $Z \gamma$ still haven't found what they are looking for (2018) arXiv:1806.09640 [hep-ph]

110. J. Kalinowski, P. Kozów, S. Pokorski, J. Rosiek, M. Szleper, S. Tkaczyk, Same-sign WW scattering at the LHC: can we discover BSM effects before discovering new states? Eur. Phys. J. C 78(5), 403 (2018). arXiv: 1802.02366 [hep-ph]

111. C. Zhang, S.-Y.Zhou, Positivity bounds on vector boson scattering at the LHC (2018) arXiv:1808.00010 [hep-ph]

112. R.L. Delgado, A. Dobado, D. Espriu, C. Garcia-Garcia, M.J. Herrero, X. Marcano, J.J. Sanz-Cillero, Production of vector resonances at the LHC via WZ-scattering: a unitarized $\mathrm{EChL}$ analysis. JHEP 11, 098 (2017). arXiv:1707.04580 [hep-ph]

113. E. Arganda, C. Garcia-Garcia, M. J. Herrero, Probing the Higgs self-coupling through double Higgs production in vector boson scattering at the LHC (2018). arXiv:1807.09736 [hep-ph]

114. J. Alwall, R. Frederix, S. Frixione, V. Hirschi, F. Maltoni, O. Mattelaer, H.S. Shao, T. Stelzer, P. Torrielli, M. Zaro, The automated computation of tree-level and next-to-leading order differential cross sections, and their matching to parton shower simulations. JHEP 07, 079 (2014). arXiv:1405.0301 [hep-ph]

115. A. Alloul, N .D. Christensen, C. Degrande, C. Duhr, B. Fuks, FeynRules 2.0 - a complete toolbox for tree-level phenomenology. Comput. Phys. Commun. 185, 2250-2300 (2014). arXiv: 1310.1921 [hep-ph]

116. E. Conte, B. Fuks, G. Serret, MadAnalysis 5, a user-friendly framework for collider phenomenology. Comput. Phys. Commun. 184, 222-256 (2013). arXiv:1206.1599 [hep-ph]

117. L. Berthier, M. Bjørn, M. Trott, Incorporating doubly resonant $W^{ \pm}$data in a global fit of SMEFT parameters to lift flat directions. JHEP 09, 157 (2016). arXiv:1606.06693 [hep-ph]

118. I. Brivio, M. Trott, Scheming in the SMEFT... and a reparameterization invariance!. JHEP 07, 148 (2017). arXiv:1701.06424 [hep-ph]. [Addendum: JHEP05,136(2018)]

119. C. Englert, R. Kogler, H. Schulz, M. Spannowsky, Higgs coupling measurements at the LHC. Eur. Phys. J. C 76(7), 393 (2016). arXiv:1511.05170 [hep-ph]
120. C. Englert, R. Kogler, H. Schulz, M. Spannowsky, Higgs characterisation in the presence of theoretical uncertainties and invisible decays. Eur. Phys. J. C 77(11), 789 (2017). arXiv:1708.06355 [hep-ph]

121. J. de Blas, M. Ciuchini, E. Franco, S. Mishima, M. Pierini, L. Reina, L. Silvestrini, The global electroweak and Higgs fits in the LHC era. PoS EPS-HEP 2017, 467 (2017). arXiv:1710.05402 [hep-ph]

122. J. Ellis, C.W. Murphy, V. Sanz, T. You, Updated global SMEFT fit to Higgs. Diboson and electroweak data. JHEP 06, 146 (2018). arXiv:1803.03252 [hep-ph]

123. S. Alioli, W. Dekens, M. Girard, E. Mereghetti, NLO QCD corrections to SM-EFT dilepton and electroweak Higgs boson production, matched to parton shower in POWHEG (2018). arXiv:1804.07407 [hep-ph]

124. J. Brehmer, A. Freitas, D. Lopez-Val, T. Plehn, Pushing Higgs effective theory to its limits. Phys. Rev. D 93(7), 075014 (2016). arXiv: 1510.03443 [hep-ph]

125. A. Biekötter, J. Brehmer, T. Plehn, Extending the limits of Higgs effective theory. Phys. Rev. D 94(5), 055032 (2016). arXiv:1602.05202 [hep-ph]

126. C. Hays, A. Martin, V. Sanz, J. Setford, On the impact of dimension-eight SMEFT operators on Higgs measurements (2018). arXiv:1808.00442 [hep-ph]

127. A. Denner, S. Pozzorini, One loop leading logarithms in electroweak radiative corrections. 1. Results. Eur. Phys. J. C 18, 461480 (2001). arXiv:hep-ph/0010201 [hep-ph]

128. B. Biedermann, A. Denner, S. Dittmaier, L. Hofer, B. Jäger, Electroweak corrections to $p p \rightarrow \mu^{+} \mu^{-} e^{+} e^{-}+X$ at the LHC: a Higgs background study. Phys. Rev. Lett. 116(16), 161803 (2016). arXiv:1601.07787 [hep-ph]

129. B. Biedermann, A. Denner, M. Pellen, Complete NLO corrections to $\mathrm{W}^{+} \mathrm{W}^{+}$scattering and its irreducible background at the LHC. JHEP 10, 124 (2017). arXiv:1708.00268 [hep-ph]

130. D. Liu, A. Pomarol, R. Rattazzi, F. Riva, Patterns of strong coupling for LHC searches. JHEP 11, 141 (2016). arXiv:1603.03064 [hep-ph]

131. O. Domenech, A. Pomarol, J. Serra, Probing the SM with Dijets at the LHC. Phys. Rev. D 85, 074030 (2012). arXiv:1201.6510 [hep-ph]

132. S. Alte, M. König, W. Shepherd, Consistent searches for SMEFT effects in non-resonant Dijet events. JHEP 01, 094 (2018). arXiv:1711.07484 [hep-ph]

133. CMS Collaboration, A. M. Sirunyan et al., Measurement of differential cross sections for $\mathrm{Z}$ boson pair production in association with jets at $\sqrt{s}=8$ and $13 \mathrm{TeV}$ (2018). arXiv:1806.11073 [hepex]

134. R. Gomez-Ambrosio, Vector boson scattering studies in CMS: the $p p \rightarrow Z Z j j$ channel. Acta Phys. Polon. Supp. 11, 239-248 (2018). arXiv:1807.09634 [hep-ph]

135. ATLAS Collaboration, T. A. collaboration, Observation of electroweak $W^{ \pm} Z$ boson pair production in association with two jets in pp collisions at $\sqrt{s}=13 \mathrm{TeV}$ with the ATLAS Detector (2018)

136. A. Ballestrero, E. Maina, G. Pelliccioli, $W$ boson polarization in vector boson scattering at the LHC. JHEP 03, 170 (2018). arXiv:1710.09339 [hep-ph] 